\title{
Recursion Operators and Bi-Hamiltonian Structures in Multidimensions. II
}

\author{
A. S. Fokas and P. M. Santini* \\ Department of Mathematics and Computer Science and Institute for Nonlinear Studies, Clarkson \\ University, Potsdam, NY 13676, USA
}

\begin{abstract}
We analyze further the algebraic properties of bi-Hamiltonian systems in two spatial and one temporal dimensions. By utilizing the Lie algebra of certain basic (starting) symmetry operators we show that these equations possess infinitely many time dependent symmetries and constants of motion. The master symmetries $\tau$ for these equations are simply derived within our formalism. Furthermore, certain new functions $T_{12}$ are introduced, which algorithmically imply recursion operators $\Phi_{12}$. Finally the theory presented here and in a previous paper is both motivated and verified by regarding multidimensional equations as certain singular limits of equations in one spatial dimension.
\end{abstract}

\section{Introduction}

This paper investigates certain algebraic aspects of exactly solvable evolution equations in $2+1$ (i.e. in two spatial and in one temporal dimensions). It is a continuation of [1], although it can be read independently.

We consider evolution equations in the form

$$
q_{t}=K(q),
$$

where $q(x, y, t)$ is an element of a suitable space $S$ of functions vanishing rapidly for large $x, y$. Let $K$ be a differentiable map on this space and assume that it does not depend explicitly on $x, y, t$. If Eq. (1.1) is integrable then it belongs to some hierarchy (generated by a recursion operator $\Phi_{12}$ ), hence in association with (1.1) we shall study $q_{t}=K^{(n)}(q)$. Fundamental in our theory is to write these equations in the form

$$
q_{1_{t}}=\int_{\mathbb{R}} d y_{2} \delta_{12} \Phi_{12}^{n} \hat{K}_{12}^{0} \cdot 1 \doteqdot \int_{\mathbb{R}} d y_{2} \delta_{12} K_{12}^{(n)}=K_{11}^{(n)}
$$

where $\delta_{12}=\delta\left(y_{1}-y_{2}\right)$ denotes the Dirac delta function, $q_{i} \doteqdot q\left(x, y_{i}, t\right), i=1,2$,

\footnotetext{
* Permanent Address: Dipartimento di Fisica. Universita di Roma, La Sapienza, J-00185 Roma, Italy
} 
$K_{12}^{(n)}\left(q_{1}, q_{2}\right)$ belong to a suitably extended space $\tilde{S}, \Phi_{12}, \hat{K}_{12}^{0}$ are operator valued functions in $\widetilde{S}$. If $q$ is a matrix function then 1 is replaced by the identity matrix. Throughout this paper $m$ and $n$ are non-negative integers.

The following results were obtained in [1]: i) There is an algorithmic approach for obtaining the recursion operator $\Phi_{12}$ from the associated isospectral eigenvalue problem. ii) This operator is hereditary. iii) Each member of the hierarchy $\left(\Phi_{12}^{m} \hat{K}_{12}^{0} \cdot 1\right)_{11} \doteqdot \int d y_{2} \delta_{12} \Phi_{12}^{m} \hat{K}_{12}^{0} \cdot 1$, where $K_{12}^{0} \cdot 1$ is a starting symmetry, is a symmetry of (1.2). For example the Kadomtsev-Petviashvili (KP) equation and the Davey-Stewartson (DS) equation admit two such hierarchies of commuting symmetries. iv) If the hereditary operator admits a factorization in terms of two Hamiltonian operators, then hierarchies of commuting symmetries give rise to hierarchies of constants of motion in involution with respect to two different Poisson brackets. For example, the KP and the DS equations admit two such hierarchies of conserved quantities.

The above results extend the theory of [2-4] to equations in $2+1$. Novel aspects of the theory in $2+1$ include: i) The role of the Frechét derivative is now played by a certain directional derivative. If subscripts $f$ and $d$ denote these derivatives then there is a simple relationship between directional and total Frechét derivatives:

$$
K_{12_{d}}\left[\delta_{12} F_{12}\right]=K_{12_{f}}[F] \doteqdot K_{12_{q_{1}}}\left[F_{11}\right]+K_{12_{q_{2}}}\left[F_{22}\right],
$$

where $K_{12}$ is an arbitrary function in $\tilde{S}$, and $K_{12 q_{i}}$ denotes the Frechét derivative of $K_{12}$ with respect to $q_{i}$, i.e.

$$
\left.K_{1 q_{q_{i}}}\left[F_{i i}\right] \doteqdot \frac{\partial}{\partial \varepsilon} K_{12}\left(q_{i}+\varepsilon F_{i i}, q_{j}\right)\right|_{\varepsilon=0}, \quad i, j=1,2, \quad i \neq j .
$$

Operators on which directional derivatives are defined are called admissible [1] (applications of the $d$-derivative in explicit examples can be found in Appendix A, see also Appendix $C$ of [1]). ii) The starting symmetry $K_{12}^{0}$ can be written as $\hat{K}_{12}^{0} \cdot 1$, where $\hat{K}_{12}^{0}$ is an admissible operator. Essential to our theory is that the operators $\hat{K}_{12}^{0}$, acting on suitable functions $H_{12}$, form a Lie algebra.

1. For the equations associated with the KP equation,

$$
\Phi_{12}=D^{2}+q_{12}^{+}+D q_{12}^{+} D^{-1}+q_{12}^{-} D^{-1} q_{12}^{-} D^{-1}, \quad q_{12}^{ \pm} \doteqdot q_{1} \pm q_{2}+\alpha\left(D_{1} \mp D_{2}\right),
$$

where $D_{i} \doteqdot \partial / \partial y_{i}$. The starting operators $\hat{K}_{12}^{0}$ are given by

$$
\hat{N}_{12} \doteqdot q_{12}^{-}, \quad \hat{M}_{12} \doteqdot D q_{12}^{+}+q_{12}^{-} D^{-1} q_{12}^{-} \text {, }
$$

and $H_{12}$ is an arbitrary function independent of $x$, i.e.

$$
H_{12}=H_{12}\left(y_{1}, y_{2}\right) \text {. }
$$

The Lie algebra of $\hat{K}_{12}^{0}$ is given by

$$
\begin{aligned}
{\left[\hat{N}_{12} H_{12}^{(1)}, \hat{N}_{12} H_{12}^{(2)}\right]_{d} } & =-\hat{N}_{12} H_{12}^{(3)},\left[\hat{N}_{12} H_{12}^{(1)}, \hat{M}_{12} H_{12}^{(2)}\right]_{d}=-\hat{M}_{12} H_{12}^{(3)}, \\
{\left[\hat{M}_{12} H_{12}^{(1)}, \hat{M}_{12} H_{12}^{(2)}\right]_{d} } & =-\Phi_{12} \hat{N}_{12} H_{12}^{(3)},
\end{aligned}
$$


where

$$
\begin{gathered}
{\left[K_{12}^{(1)}, K_{12}^{(2)}\right]_{d}=K_{12_{d}}^{(1)}\left[K_{12}^{(2)}\right]-K_{12_{d}}^{(2)}\left[K_{12}^{(1)}\right],} \\
H_{12}^{(3)} \doteqdot\left[H_{12}^{(1)}, H_{12}^{(2)}\right]_{I} \doteqdot \int_{\mathbb{Q}} d y_{3}\left(H_{13}^{(1)} H_{32}^{(2)}-H_{13}^{(2)} H_{32}^{(1)}\right) .
\end{gathered}
$$

2. For the equations associated with the DS equation

$$
\begin{aligned}
\Phi_{12} & =\sigma\left(P_{12}-Q_{12}^{+} P_{12}^{-1} Q_{12}^{+}\right), \quad Q_{12}^{ \pm} F_{12} \doteqdot Q_{1} F_{12} \pm F_{12} Q_{2}, \\
P_{12} F_{12} & \doteqdot F_{12 x}-J F_{12_{v_{1}}}-F_{12_{y_{2}}} J,
\end{aligned}
$$

where $J=\alpha \sigma, \sigma=\operatorname{diag}(1,-1), Q$ is a $2 \times 2$ off-diagonal matrix containing the potentials $q_{1}(x, y), q_{2}(x, y)$ and $\Phi_{12}$ is defined on off-diagonal matrices. The starting operators $\hat{K}_{12}^{0}$ are given by:

$$
\hat{N}_{12} \doteqdot Q_{12}^{-}, \quad \hat{M}_{12} \doteqdot Q_{12}^{-} \sigma
$$

and $H_{12}$ is an arbitrary matrix function satisfying the following properties:

$$
H_{12} \text { diagonal matrix, } P_{12} H_{12}=0 \text {. }
$$

Also

$$
\begin{aligned}
{\left[\hat{N}_{12} H_{12}^{(1)}, \hat{N}_{12} H_{12}^{(2)}\right]_{d} } & =-\hat{N}_{12} H_{12}^{(3)}, \quad\left[\hat{N}_{12} H_{12}^{(1)}, \hat{M}_{12} H_{12}^{(2)}\right]_{d}=-\hat{M}_{12} H_{12}^{(3)}, \\
{\left[\hat{M}_{12} H_{12}^{(1)}, \hat{M}_{12} H_{12}^{(2)}\right]_{d} } & =-\hat{N}_{12} H_{12}^{(3)} .
\end{aligned}
$$

iii) The recursion operator $\Phi_{12}$ is admissible and enjoys a simple commutator operator relation with $h_{12}=h\left(y_{1}-y_{2}\right)$ :

$$
\left[\Phi_{12}, h_{12}\right]=-\beta h_{12}^{\prime}, \quad h_{12}^{\prime} \doteqdot \frac{\partial h_{12}}{\partial y_{1}},
$$

which implies that $\delta_{12} K_{12}^{(n)}=\delta_{12} \Phi_{12}^{n} \hat{K}_{12}^{0} \cdot 1=\sum_{l=0}^{n} \beta^{l}\left(\begin{array}{l}n \\ l\end{array}\right) \Phi_{12}^{n-l} \delta_{12}^{l} \hat{K}_{12}^{0} \cdot 1$, where $\delta_{12}^{l} \doteqdot \partial^{l} \delta_{12} / \partial y_{1}^{l}$.

The starting operator $\hat{K}_{12}^{0}$ is also admissible and its commutator relation with $h_{12}$ implies that $\delta_{12} K_{12}^{(n)}$ can be written in the form

$$
\delta_{12} K_{12}^{(n)}=\delta_{12} \Phi_{12}^{n} \hat{K}_{12}^{0} \cdot 1=\sum_{l=1}^{n} b_{n, l} \Phi_{12}^{n-l} \hat{K}_{12}^{0} \cdot \delta_{12}^{l}
$$

for suitable constants $b_{n, l}$.

1. For the two classes of evolution equations associated with the KP equation we have that

$$
\beta=-4 x,\left[\hat{N}_{12}, h_{12}\right]=0, \quad\left[\hat{M}_{12}, h_{12}\right]=-\tilde{\beta} D h_{12}^{\prime}, \quad \tilde{\beta}=\beta / 2,
$$

and

$$
b_{n, l}= \begin{cases}\beta^{l}\left(\begin{array}{l}
n \\
l
\end{array}\right), & \text { for } \hat{K}_{12}^{0}=\hat{N}_{12} \\
\sum_{s=0}^{l} \beta^{l-s} \tilde{\beta}^{s}\left(\begin{array}{l}
n-s \\
l-s
\end{array}\right), & \text { for } \hat{K}_{12}^{0}=\hat{M}_{12} .\end{cases}
$$


2. For the two classes of evolution equations associated with the DS equation we have that

and

$$
\beta=2 \alpha, \quad\left[\hat{N}_{12}, h_{12}\right]=\left[\hat{M}_{12}, h_{12}\right]=0
$$

$$
b_{n, l}=\beta^{l}\left(\begin{array}{l}
n \\
l
\end{array}\right) .
$$

In [1] we assume knowledge of the underlying isospectral problem. This problem implies: a) a hereditary operator $\Phi_{12}$ : b) suitable starting operators, say $\hat{M}_{12}$ and $\hat{N}_{12}$, and functions $H_{12}$; c) two skew symmetric operators such that $\Phi_{12}=\Theta_{12}^{(2)}\left(\Theta_{12}^{(1)}\right)^{-1}$. Furthermore, it can be shown that $\Phi_{12}$ is a strong symmetry for the starting symmetries. One then needs to: a) Find $\beta$ and $b_{n, l}$ appearing in Eqs. (1.14), (1.15). b) Compute the Lie algebras of $\hat{M}_{12}, \hat{N}_{12}$ on function $H_{12}$ (i.e. obtain equations analogous to (1.7), (1.13). c) Verify that the starting symmetries correspond to extended gradients, i.e. verify that $\left(\left(\Theta_{12}^{(1)}\right)^{-1} \hat{K}_{12}^{0} \cdot H_{12}\right)_{d}, \hat{K}_{12}^{0}=\hat{M}_{12}$ or $\hat{N}_{12}$, is symmetric with respect to the bilinear form

$$
\left\langle g_{12}, f_{12}\right\rangle \doteqdot \int_{\mathbb{R}^{3}} d x d y_{1} d y_{2} \operatorname{trace} g_{21} f_{12} \text {. }
$$

d) Verify that $\Theta_{12}^{(1)}, \Theta_{12}^{(2)}$ are compatible Hamiltonian operators.

In this paper the following results are presented. i) In Sect. 2 we investigate further the Lie algebra of the starting symmetries $\hat{K}_{12}^{0} H_{12}$. In [1] we only used a subclass of solutions of (1.6) and (1.12), given by $H_{12}=h_{12}=h\left(y_{1}-y_{2}\right)$ and $H_{12}=h_{12}(a I+b \sigma), a, b$, constants, respectively. This gave rise to time-independent commuting symmetries. We now choose $H_{12}$ to be a more general solution of the above equations; this gives rise to time dependent symmetries. Time dependent symmetries for the KP have been studied in $[6,7,18,20]$. ii) In Sect. 3, using the Lie algebra of $\hat{K}_{12}^{0} H_{12}$ and an isomorphism between Lie and Poisson brackets we prove directly that $\Phi_{12}^{n} \hat{K}_{12}^{0} H_{12}$ correspond to conserved quantities. This derivation, which capitalizes on the arbitrariness of $H_{12}$, has the advantage that does not use the bi-Hamiltonian factorization of $\Phi_{12}$. In other words, for the theory developed in this paper one needs only to verify a)-c) above.

We recall that Fuchssteiner and one of the authors (ASF) introduced an alternative way for generating symmetries, the so-called master-symmetry approach. A master-symmetry is a function $\tau$ which has the property that its Lie commutator with a symmetry is also a symmetry. The $\tau$ functions for the Benjamin-Ono and the KP equations were given in [5] and [6-7] respectively. Several authors (e.g. [8]-[12]) have noticed that master-symmetries also exist for equations in $1+1$ as well as for finite dimensional systems [13]. Let $\tau$ and $T$ denote mastery-symmetries for equations in $2+1$ and $1+1$ respectively. If $\Phi$ is the recursion operator and $\Sigma=t K+T_{0}$ is the scaling symmetry of an equation in $1+1, q_{t}=K$, then $T=\Phi T_{0}$ is a master symmetry. However, there exists a fundamental difference between $\tau$ and $T$. The function $\Theta^{-1} T(\Theta$ is a Hamiltonian operator) is not a gradient function; this can be used to constructively obtain $\Phi$ from $T$. But $\Theta^{-1} \tau$ is a gradient and hence the above construction of $\Phi$ from $\tau$ fails.

In Sect. 4 we show that $\tau$ is not the proper analogue of $T$. Let us consider the $\mathrm{KP}$ for concreteness. As it was mentioned earlier, $\Phi_{12}^{n} \hat{K}_{12}^{0} \cdot 1$ generates time- 
independent symmetries; it will be shown here that $\Phi_{12}^{n} \hat{K}_{12}^{0}\left(y_{1}+y_{2}\right)^{m}$ generates time-dependent symmetries. It turns out that $\tau=\left(\Phi_{12}^{2} \hat{K}_{12}^{0}\left(y_{1}+y_{2}\right)\right)_{11}$ (see Sect. IID). But $\Theta_{12}^{-1} \Phi_{12}^{n} \hat{K}_{12}^{0} H_{12}$ is an extended gradient for all $H_{12}$, hence $\Theta^{-1} \tau$ is a gradient function. In Sect. 4 we show that the proper analogue of $T$ for the $\mathrm{KP}$ is $T_{12} \doteqdot \Phi_{12}^{2} \delta_{12}$ (it corresponds to $\Phi^{2} \cdot 1$ for the $\mathrm{KdV}$ ). Actually, $\Theta_{12}^{-1} T_{12}$ is not an extended gradient and it can be used to constructively obtain $\Phi_{12}$.

In Sect. 5 we show that exactly solvable $2+1$ dimensional equations are exact reductions of nonlocal evolution equations generated via nonlocal isospectral eigenvalue problems. This result both motivates the basic ideas and concepts introduced in [1] and in this paper, as well as verifies several results presented in the above papers.

\section{A Lie-Algebra for Equations in $2+1$}

In developing a theory for time-dependent symmetries in $2+1$ it is useful first to: i) characterize the commutator properties of these symmetries, ii) study the action of $\Phi$ on the Lie commutator $[a, b]_{L}$, where

$$
[a, b]_{L} \doteqdot a_{L}[b]-b_{L}[a]
$$

and $a_{L}$ denotes an appropriate derivative. This derivative is linear and satisfies the Liebnitz rule. For equations in $1+1$ one only needs $[a, b]_{f}$, while for equations in $2+1$ one also needs $\left[a_{12}, b_{12}\right]_{d}$ (see (1.3)).

Lemma 2.1. $\sigma^{(r)}$ is a time dependent symmetry of order $r$ of the equation $q_{t}=K$, i.e.

$$
\frac{\partial \sigma^{(r)}}{\partial t}+\left[\sigma^{(r)}, K\right]_{L}=0
$$

iff

$$
\sigma^{(r)}=\sum_{j=0}^{r} t^{j} \Sigma^{(j)}, \quad \Sigma^{(j)} \doteqdot-\frac{1}{j}\left[\Sigma^{(j-1)}, K\right]_{L}, \quad j=1, \ldots r, \quad\left[K, \Sigma^{(r)}\right]_{L}=0 .
$$

The above result follows from the definition of a symmetry and the assumption that $\Sigma^{(j)}$ is time independent. It implies that constructing a symmetry of order $l$ is equivalent to finding a function $\Sigma^{(0)}$ with the property that its $(l+1)^{s t}$ commutator with $K$ is zero.

The action of a hereditary operator $\Phi$ on a Lie commutator is given by:

Theorem 2.1. Let

$$
S \doteqdot \Phi_{L}[K]+\left[\Phi, K_{L}\right]
$$

Then

$\left.\mathrm{a}_{1}\right) \quad \Phi^{n}\left[K_{1}, K_{2}\right]_{L}=\left[K_{1}, \Phi^{n} K_{2}\right]_{L}+\left(\sum_{r=1}^{n} \Phi^{n-r} S_{1} \Phi^{r-1}\right) K_{2}$.

If $\Phi$ is hereditary, i.e. if

$$
\Phi_{L}[\Phi v] w-\Phi \Phi_{L}[v] w \text { is symmetric with respect to } v, w,
$$


then the following are true:

a $) \Phi_{L}\left[\Phi^{n} K\right]+\left[\Phi,\left(\Phi^{n} K\right)_{L}\right]=\Phi^{n} S$,

a $) \Phi^{n+m}\left[K_{1}, K_{2}\right]_{L}=\left[\Phi^{n} K_{1}, \Phi^{m} K_{2}\right]_{L}$

$$
+\Phi^{n}\left(\sum_{r=1}^{m} \Phi^{m-r} S_{1} \Phi^{r-1}\right) K_{2}-\Phi^{m}\left(\sum_{r=1}^{n} \Phi^{n-r} S_{2} \Phi^{r-1}\right) K_{1} \text {. }
$$

( $m, n$ are non-negative integers).

Proof. To prove (2.5) use induction: $(2.5)_{0}$ is an identity. Applying $\Phi$ on $(2.5)_{n}$ we obtain

$$
\Phi^{n+1}\left[K_{1}, K_{2}\right]_{L}=\Phi\left[K_{1}, \Phi^{n} K_{2}\right]_{L}+\Phi\left(\sum_{r=1}^{n} \Phi^{n-r} S_{1} \Phi^{r-1}\right) K_{2}
$$

Equation $(2.5)_{n+1}$ follows from the above and the following identity

$$
\Phi\left[K_{1}, M\right]_{L}=\left[K_{1}, \Phi M\right]_{L}+S_{1} M .
$$

Equation (2.7) also follows from induction. To prove (2.8) first note that (2.5) implies

$$
\Phi^{m}\left[K_{1}, K_{2}\right]_{L}-\left(\sum_{r=1}^{m} \Phi^{m-r} S_{1} \Phi^{r-1}\right) K_{2}=\left[K_{1}, \Phi^{m} K_{2}\right]_{L} .
$$

Equation (2.5) also implies

$$
\Phi^{n}\left[K_{1}, \tilde{K}_{2}\right]_{L}=\left[\Phi^{n} K_{1}, \tilde{K}_{2}\right]_{L}-\left(\sum_{r=1}^{n} \Phi^{n-r} \tilde{S}_{2} \Phi^{r-1}\right) K_{1} .
$$

Let $\widetilde{K}_{2}=\Phi^{m} K_{2}$, then (2.6) implies $\widetilde{S}_{2}=\Phi^{m} S_{2}$, and the above equation becomes

$$
\Phi^{n}\left[K_{1}, \Phi^{m} K_{2}\right]_{L}=\left[\Phi^{n} K_{1}, \Phi^{m} K_{2}\right]_{L}-\left(\sum_{r=1}^{n} \Phi^{n-r} \Phi^{m} S_{2} \Phi^{r-1}\right) K_{1} .
$$

Applying $\Phi^{n}$ on (2.9) and using the above we obtain (2.8).

Corollary 2.1. Let the hereditary operator $\Phi$ be a strong symmetry for both $K_{1}$ and $K_{2}$, i.e. $S_{1}=S_{2}=0$. Then

$$
\Phi^{n+m}\left[K_{1}, K_{2}\right]_{L}=\left[\Phi^{n} K_{1}, \Phi^{m} K_{2}\right]_{L} .
$$

In the rest of this section we characterize extended symmetries $\sigma_{12}$. The following theorem, proven in [1], maps extended symmetries $\sigma_{12}$ to symmetries $\sigma_{11}$.

Theorem 2.2. Assume that the commutator of $\Phi_{12}$ with $h_{12}$ is given by (1.14) and that the starting operator $\hat{K}_{12}^{0}$ are such that $(1.15)$ is valid. If $\sigma_{12}$ is an extended symmetry of (1.2), i.e. if

$$
\frac{\partial \sigma_{12}}{\partial t}+\left[\sigma_{12}, \delta_{12} \Phi_{12}^{n} \hat{K}_{12}^{0} \cdot 1\right]_{d}=0
$$

then $\sigma_{11}$ is a symmetry of (1.2), i.e. 


$$
\frac{\partial \sigma_{11}}{\partial t}+\left[\sigma_{11}, K_{11}^{(n)}\right]_{f}=0
$$

In the above

and

$$
\left[\sigma_{11}, K_{11}^{(n)}\right]_{f}=\sigma_{1 q_{q_{1}}}\left[K_{11}^{(n)}\right]-K_{11_{q_{1}}}^{(n)}\left[\sigma_{11}\right]
$$

$$
\left[\sigma_{12}, \delta_{12} \Phi_{12}^{n} \hat{K}_{12}^{0} \cdot 1\right]_{d}=\sum_{l=0}^{n} b_{n, l}\left[\sigma_{12}, \Phi_{12}^{n-l} \hat{K}_{12}^{0} \delta_{12}^{l}\right]_{d}
$$

It is necessary to rewrite $\delta_{12} \Phi_{12}^{n} \hat{K}_{12}^{0} \cdot 1$ in the form appearing in (2.14) since the directional derivative is defined only for functions of the form $\hat{L}_{12} H_{12}$, where $\hat{L}_{12}$ is an admissible operator.

Using Lemma 2.1, Corollary 2.1 and the Lie algebra of $\hat{K}_{12}^{0} H_{12}$ (with appropriate $H_{12}$ ) we obtain extended symmetries, which then via Theorem 2.2 give rise to symmetries.

Proposition 2.1. Assume that the hereditary operator $\Phi_{12}$ is a strong symmetry for the admissible starting operators $\hat{M}_{12}, \hat{N}_{12}$, and that (1.14), (1.15) hold. Further assume that $\hat{M}_{12}, \hat{N}_{12}$ form a Lie algebra (analogous to (1.7), (1.13)). Consider the following hierarchies

$$
\begin{aligned}
& q_{1_{t}}=\int_{\mathbb{R}} d y_{2} \delta_{12} \Phi_{12}^{n} \hat{N}_{12} \cdot 1=\int_{\mathbb{R}} d y_{2} \delta_{12} N_{12}^{(n)}=N_{11}^{(n)}, \\
& q_{1_{t}}=\int_{\mathbb{R}} d y_{2} \delta_{12} \Phi_{12}^{n} \hat{M}_{12} \cdot 1=\int_{\mathbb{R}} d y_{2} \delta_{12} M_{12}^{(n)}=M_{11}^{(n)} .
\end{aligned}
$$

Then:

a) $\left(\Phi_{12}^{m} \hat{M}_{12} \cdot 1\right)_{11},\left(\Phi_{12}^{m} \hat{N}_{12} \cdot 1\right)_{11}$, are symmetries of Eqs. (2.15).

b) Appropriate linear combinations of $\left\{\Phi_{12}^{m} \hat{M}_{12} H_{12}^{(r)}\right\}_{11},\left\{\Phi_{12}^{m} \hat{N}_{12} H_{12}^{(r)}\right\}_{11}$ for suitable functions $H_{12}^{(r)}$ generate time dependent symmetries for Eqs. (2.15).

Rather than proving the above proposition in general, we use for concreteness, the Lie algebra (1.6) to sketch how the above results can be derived. Details are given in II.A, II.B. Let

$$
\hat{N}_{12}^{(n)} \doteqdot \Phi^{n} \hat{N}_{12}, \quad \hat{M}_{12}^{(n)} \doteqdot \Phi^{n} \hat{M}_{12} .
$$

Then, using Corollary 2.1, Eqs. (1.7) imply

$$
\begin{aligned}
{\left[\hat{N}_{12}^{(m)} H_{12}^{(1)}, \hat{N}_{12}^{(n-l)} H_{12}^{(2)}\right]_{d} } & =-\hat{N}^{(m+n-l)} H_{12}^{(3)}, \\
{\left[\hat{N}_{12}^{(m)} H_{12}^{(1)}, \hat{M}_{12}^{(n-l)} H_{12}^{(2)}\right]_{d} } & =-\hat{M}_{12}^{(m+n-l)} H_{12}^{(3)}, \\
{\left[\hat{M}_{12}^{(m)} H_{12}^{(1)}, \hat{N}_{12}^{(n-l)} H_{12}^{(2)}\right]_{d} } & =-\hat{M}_{12}^{(m+n-l)} H_{12}^{(3)}, \\
{\left[\hat{M}_{12}^{(m)} H_{12}^{(1)}, \hat{M}_{12}^{(n-l)} H_{12}^{(2)}\right]_{d} } & =-\hat{N}_{12}^{(m+n+1-l)} H_{12}^{(3)} .
\end{aligned}
$$

Part a) of the proposition is a direct consequence of Eqs. (2.17) and (2.14). For example

$$
\left[\hat{N}_{12}^{(m)} \cdot 1, \delta_{12} \hat{N}_{12}^{(n)} \cdot 1\right]_{d}=-\sum_{l=0}^{n} b_{n, l} \hat{N}^{(m+n-l)} \cdot \tilde{H}_{12}^{(l)}=0,
$$

since $\tilde{H}_{12}^{(l)}=\left[1, \delta_{12}^{l}\right]_{I}=0$; thus $\hat{N}_{12}^{(m)} \cdot 1$ are extended symmetries of $(2.15 \mathrm{a})$.

Consider part b) of Proposition 2.1. Let us first consider symmetries of order 
one it $t$. Then

$$
\begin{aligned}
& \hat{N}_{12}^{(m)}\left(y_{1}+y_{2}\right)-t 2 \beta\left(\begin{array}{l}
n \\
1
\end{array}\right) \hat{N}_{12}^{(m+n-1)} \cdot 1, \\
& \hat{M}_{12}^{(m)}\left(y_{1}+y_{2}\right)-t 2 \beta\left(\begin{array}{l}
n \\
1
\end{array}\right) \hat{M}_{12}^{(m+n-1)} \cdot 1
\end{aligned}
$$

are first order time dependent extended symmetries of (2.15a). Similarly

$$
\begin{aligned}
& \hat{N}_{12}^{(m)} \cdot\left(y_{1}+y_{2}\right)-t 2 b_{n, 1} \hat{M}_{12}^{(n+m-1)} \cdot 1, \\
& \hat{M}_{12}^{(m)} \cdot\left(y_{1}+y_{2}\right)-t 2 b_{n, 1} \hat{N}^{(m+n)} \cdot 1,
\end{aligned}
$$

are extended symmetries of $(2.15 b)$ with $b_{n, l}=(-4 \alpha) \sum_{s=0}^{1} 2^{-s}\left(\begin{array}{c}n-s \\ l-s\end{array}\right)$.

To derive the above we use Lemma 2.1 and Eqs. (2.17). For example, to derive (2.18) we look for a function $\Sigma_{12}^{(0)}$ such that its commutator with $\delta_{12} \hat{N}_{12}^{(n)} \cdot 1$, commutes with $\delta_{12} \hat{N}_{12}^{(n)} \cdot 1$. Clearly $\Sigma_{12}^{(0)}=\hat{N}_{12}^{(m)}\left(y_{1}+y_{2}\right)$ or $\hat{M}_{12}^{(m)}\left(y_{1}+y_{2}\right)$. For, (2.17a) implies

$$
\left[\widehat{N}_{12}^{(m)}\left(y_{1}+y_{2}\right), \delta_{12} \hat{N}_{12}^{(n)} \cdot 1\right]_{d}=2 \beta\left(\begin{array}{l}
n \\
1
\end{array}\right) \hat{N}^{(m+n-1)} \cdot 1
$$

since $\tilde{H}_{12}^{(l)}=\left[y_{1}+y_{2}, \delta_{12}^{l}\right]_{I}=-2 \delta_{1, l}$, where $\delta_{1 . l}=0$ if $l \neq 1$ or 1 if $l=1$.

In a similar manner

$$
\begin{aligned}
& \hat{N}_{12}^{(m)}\left(y_{1}+y_{2}\right)^{2}-t 4 \beta\left(\begin{array}{l}
n \\
1
\end{array}\right) \hat{N}_{12}^{(m+n-1)}\left(y_{1}+y_{2}\right)-t^{2} 4 \beta^{2}\left(\begin{array}{c}
n \\
1
\end{array}\right)^{2} \hat{N}_{12}^{(m+2 n-2)} \cdot 1, \\
& \hat{M}_{12}^{(m)}\left(y_{1}+y_{2}\right)^{2}-t 4 \beta\left(\begin{array}{l}
n \\
1
\end{array}\right) \hat{M}_{12}^{(m+n-1)}\left(y_{1}+y_{2}\right)+t^{2} 4 \beta^{2}\left(\begin{array}{c}
n \\
1
\end{array}\right)^{2} \hat{M}_{12}^{(m+2 n-2)} \cdot 1
\end{aligned}
$$

are second order time dependent extended symmetries of (2.15b). Similarly

$$
\begin{aligned}
& \hat{N}^{(m)} \cdot\left(y_{1}+y_{2}\right)^{2}-t 4 b_{n, 1} \hat{M}^{(m+n-1)} \cdot\left(y_{1}+y_{2}\right)+t^{2} 4 b_{n, 1}^{2} \hat{N}^{(m+2 n-1)} \cdot 1, \\
& \hat{M}^{(m)} \cdot\left(y_{1}+y_{2}\right)^{2}-t 4 b_{n, 1} \hat{N}^{(m+n)} \cdot\left(y_{1}+y_{2}\right)+t^{2} 4 b_{n, 1}^{2} \hat{M}^{(m+2 n-1)} \cdot 1, \\
& \quad b_{n, 1}=(-4 \alpha)\left(n+\frac{1}{2}\right)
\end{aligned}
$$

are extended symmetries of $(2.15 \mathrm{~b})$. Indeed

$$
\left[\hat{N}_{12}^{(m)}\left(y_{1}+y_{2}\right)^{2}, \delta_{12} \hat{N}_{12}^{(n)} \cdot 1\right]_{d}=4 \beta\left(\begin{array}{l}
n \\
1
\end{array}\right) \hat{N}_{12}^{(m+n-1)}\left(y_{1}+y_{2}\right),
$$

since, $\left[\left(y_{1}+y_{2}\right)^{2}, \delta_{12}^{l}\right]=-4\left(y_{1}+y_{2}\right) \delta_{1, l}$. Also

$$
\left[\hat{N}_{12}^{(m+n-1)}\left(y_{1}+y_{2}\right), \delta_{12} \hat{N}_{12}^{(n)} \cdot 1\right]=2 \beta\left(\begin{array}{l}
n \\
1
\end{array}\right) \hat{N}_{12}^{(m+2 n-2)} .
$$

The extension of the above results to any order in time is straightforward: To generate $\sigma_{12}^{(r)}$ consider $\Sigma_{12}^{(0)}=\hat{N}_{12}^{(m)}\left(y_{1}+y_{2}\right)^{r}$ or $\hat{M}_{12}^{(m)}\left(y_{1}+y_{2}\right)^{r}$. The commutator of $\left(y_{1}+y_{2}\right)^{r}$ with $\delta_{12}^{l}$ produces $\left(y_{1}+y_{2}\right)^{r-l}$. Thus the $r^{\text {th }}$ commutator of $\left(y_{1}+y_{2}\right)^{r}$ 
with $\delta_{12}^{l}$ produces 1 which commutes with $\delta_{12}^{(l)}$; hence Lemma 2.1 guarantees the existence of an $r^{\text {th }}$ order symmetry.

II.A. Time Dependent Symmetries for the Equations Associated with the KP Equation. Following the construction and the argument sketched above, extended symmetries of order $r$ in time

$$
\sigma_{12}^{(r)}=\sum_{j=0}^{r} t^{j} \Sigma_{12}^{(j)}
$$

are generated through Proposition 2.1, starting with $\Sigma_{12}^{(0)}=\hat{N}_{12}^{(m)} \cdot H_{12}^{(r)}$ or $\hat{M}_{12}^{(m)} \cdot H_{12}^{(r)}$, where $H_{12}^{(r)}$, is defined by

$$
H_{12}^{(r)} \doteqdot\left(y_{1}+y_{2}\right)^{r}
$$

more generally, any homogeneous polynomial of degree $r$ in $y_{1}$ and $y_{2}$ could be used as well (note $H_{12}^{r}$ solves (1.6)). Using

$$
\begin{gathered}
{\left[H_{12}^{(r)}, \delta_{12}^{s}\right]=-\left(1-(-1)^{s}\right) \theta(r-s) \frac{r !}{(r-s) !} H_{12}^{(r-s)},} \\
\theta(a)= \begin{cases}1, & a \geqq 0, \\
0, & a<0,\end{cases}
\end{gathered}
$$

we can show that

i) The class of evolution equations (2.15a) with $\hat{N}_{12}=q_{12}^{-}$admits $t$-dependent symmetries of order $r$ given by

$$
\begin{aligned}
& \Sigma_{12}^{(0)}=\hat{N}_{12}^{(m)} \cdot H_{12}^{(r)}, \\
& \Sigma_{12}^{(j)}=\Sigma v(r, j, s) \hat{N}_{12}^{\left(m+j n-\sum_{l=1}^{j} 2 s_{l}+1\right)} \cdot H_{12}^{\left(r-\sum_{l=1}^{j} 2 s_{l}+1\right)},
\end{aligned}
$$

and by

$$
\begin{aligned}
& \Sigma_{12}^{(0)}=\hat{M}_{12}^{(m)} \cdot H_{12}^{(r)}, \\
& \Sigma_{12}^{(j)}=\Sigma v(r, j, s) \hat{M}^{\left(m+j-\sum_{l=1}^{j} 2 s_{l}+1\right)} \cdot H_{12}^{\left(r-\sum_{l=1}^{j} 2 s_{l}+1\right)},
\end{aligned}
$$

where $j \geqq 1$, the summation $\Sigma$ is from $s_{1}, s_{2}, \ldots, s_{j}$ zero to $P_{n}$ and $P_{n}=(n-1) / 2$ if $n$ is odd and $(n-2) / 2$ if $n$ is even. Also

$$
v(r, j, s) \doteqdot \frac{(-2)^{j}}{j !}\left(\prod_{\mu=1}^{j} \theta\left(r-\sum_{l=1} 2 s+1\right)\right)\left(\prod_{l=1}^{j} b_{n, 2 s_{l}+1}\right) \frac{r !}{\left(r-\sum_{l=1}^{j} 2 s_{l}+1\right) !}
$$

and $b_{n, l}=(-4 \alpha)^{l}\left(\begin{array}{l}n \\ l\end{array}\right)$.

ii) The KP class $(2.15 \mathrm{~b})$ with $\hat{M}_{12}=D q_{12}^{+}=q_{12}^{-} D^{-1} q_{12}^{-}$admits $t$-dependent symmetries of order $r$ given by

$$
\Sigma_{12}^{(0)}=\hat{N}_{12}^{(m)} \cdot H_{12}^{(r)},
$$




$$
\begin{gathered}
\Sigma_{12}^{(2 j)}=\Sigma v(r, 2 j, s) \hat{N}_{12}^{\left(m+2 j n+\jmath-\sum_{l=1}^{2 l} 2 s_{l}+1\right)} \cdot H_{12}^{\left(r-\sum_{l=1}^{2 j} 2 s_{l}+1\right)}, \\
\Sigma_{12}^{(2 j-1)}=\Sigma v(r, 2 j-1, s) \hat{M}_{12}^{\left(m+(2 j-1) n+\jmath-1-\sum_{l=1}^{2 l-1} 2 s_{l}+1\right)} \cdot H_{12}^{\left(r-\sum_{l=1}^{2 j-1} 2 s_{l}+1\right)},
\end{gathered}
$$

and by

$$
\begin{aligned}
\Sigma_{12}^{(0)} & =\hat{M}_{12}^{(m)} \cdot H_{12}^{(r)}, \\
\Sigma_{12}^{(2 j)} & =\Sigma v(r, 2 j, s) \hat{M}_{12}^{\left(m+2 j n+1-\sum_{l=1}^{2 j} 2 s_{l}+1\right)} \cdot H_{12}^{\left(r-\sum_{l=1}^{2 j} 2 s_{l}+1\right)}, \\
\Sigma_{12}^{(2 j-1)} & =\Sigma v(r, 2 j-1, s) \hat{N}_{12}^{\left(m+(2 j-1) n+j-\sum_{l=1}^{2 J-1} 2 s_{l}+1\right)} \cdot H_{12}^{\left(r-\sum_{l=1}^{2 J-1} 2 s_{l}+1\right)},
\end{aligned}
$$

with $j \geqq 1$ and $b_{n, l}=\sum_{s=0}^{l} \beta^{l-s} \widetilde{\beta}^{s}\left(\begin{array}{c}n-s \\ l-s\end{array}\right)=(-4 \alpha)^{l} \sum_{s=0}^{l} 2^{-s}\left(\begin{array}{l}n-s \\ l-s\end{array}\right)$.

II.B. Time Dependent Symmetries for the Equations Associated with the DaveyStewartson Equation. The construction of $t$-dependent symmetries for the equations associated with the DS equation is similar. Extended symmetries of order $r$ in time are generated through Lemma 2.1, starting with $\Sigma_{12}^{(0)}=\hat{N}^{(m)} H_{12}^{(r)}$ or $\hat{M}_{12}^{(m)} H_{12}^{(r)}$, where $H_{12}^{(r)}$ is defined by,

$$
H_{12}^{(r)} \doteqdot \operatorname{diag}\left(\xi_{+_{12}}^{r}, \xi_{-12}^{r}\right), \quad \xi_{ \pm_{12}} \doteqdot y_{1}+y_{2} \pm 2 x x .
$$

$H_{12}^{(r)}$ satisfies the same formula (2.24), obviously replacing $\left[H_{12}^{(r)}, \delta_{12}^{s}\right]_{I}$ by $\left[H_{12}^{(r)}\right.$, $\left.\delta_{12}^{s} I\right]_{I}$. Then, using Corollary 2.1 and Eqs. (1.13), one can show that

i) The class of evolution equations (2.15a) with $\hat{N}_{12}=Q_{12}^{-}$admits $t$-dependent symmetries of order $r$ given by Eqs. (2.26) and (2.27), where $b_{n, l}=\beta^{l}\left(\begin{array}{l}n \\ l\end{array}\right)=(2 \alpha)^{l}\left(\begin{array}{l}n \\ l\end{array}\right)$ and $j \geqq 1$.

ii) The class of evolution equations (2.15b) with $\hat{M}_{12}=Q_{12}^{-} \sigma$ admits $t$ dependent symmetries of order $r$ given by Eqs. $(2.29-30)$, replacing: $\hat{N}^{(\cdot)} \rightarrow \widehat{N}^{(-j)}$ in Eq. (2.29b), $\hat{M}^{(\cdot)} \rightarrow \hat{M}^{(\cdot-j+1)}$ in Eq. (2.29c), $\hat{M}^{(\cdot)} \rightarrow \hat{M}^{(\cdot-j)}$ in Eq. (2.30b), $\hat{N}^{(\cdot)} \rightarrow \hat{N}^{(\cdot-j)}$ in Eq. $(2.30 \mathrm{c})$ and using $b_{n, l}=(2 \alpha)^{l}\left(\begin{array}{l}n \\ l\end{array}\right)$.

II.C. Connection with Known Results. Before the discovery [14] of the recursion operator of the KP equation, a different approach, the so-called master-symmetries approach, was used to generate an infinite sequence of commuting symmetries [6], as well as $t$-dependent symmetries [7-11], of the KP equation (see also $[18,19]$ ).

The existence of a hereditary operator in $2+1$ dimensions, together with the Lie algebra of the starting symmetries allows a simple and elegant characterization of the $2+1$ dimensional (gradient) master-symmetries introduced in the above papers. Here we briefly consider the KP example. 
In Proposition 2.1 and in Sect. II.B. we have shown that the functions

$$
\tau_{12}^{(m, r)} \doteqdot \Phi_{12}^{m} K_{12}^{0} H_{12}^{(r)}
$$

(where $H_{12}^{(r)}$ is defined in (2.23), but it could be any homogeneous polynomial of degree $r$ in $y_{1}, y_{2}$, and $\hat{K}_{12}^{0}$ is $\hat{N}_{12}$ or $\left.\hat{M}_{12}\right)$ have the property that their $(r+1)^{s t}$ commutator with $\delta_{12} K_{12}^{(n)}$ is zero, namely

$$
\underbrace{\left[\cdots\left[\tau_{12}^{(m, r)}, \delta_{12} K_{12}^{(n)}\right]_{d} \cdots\right]_{d+1 \text { times }}}_{r+1 \text { times }}=0 .
$$

Then Theorem 4.1 of [1] implies that

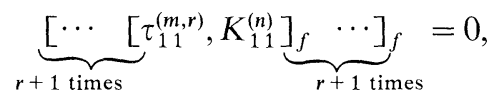

namely $\tau_{11}^{(m, r)}$ are the so-called master-symmetries of degree $r$ of KP [11]. Equation (2.33) essentially follows from the fact that a single commutator of $\tau_{12}^{(m, r)}$ with $\delta_{12} K_{12}^{(n)}$ generates a linear combination of lower degree master-symmetries; in fact, choosing for concreteness $\tau_{12}^{(m, r)}=\Phi_{12}^{m} \hat{N}_{12}\left(y_{1}+y_{2}\right)^{r}$ and $K_{12}^{(n)}=M_{12}^{(n)}$, we have

$$
\begin{aligned}
{\left[\tau_{12}^{(m, r)}, \delta_{12} M_{12}^{(n)}\right]_{d} } & =-\sum_{l=0}^{n} b_{n, l} \hat{M}_{12}^{(m+n)}\left[\left(y_{1}+y_{2}\right)^{r}, \delta_{12}^{l}\right] \\
& =\sum_{l=1}^{n} \theta(r-l) \frac{r !}{(r-l) !} b_{n, l} \tau_{12}^{(m+n, r-l)},
\end{aligned}
$$

which implies

$$
\left[\tau_{11}^{(m, r)}, M_{11}^{(n)}\right]_{f}=\sum_{l=1}^{n} \theta(r-l) \frac{r !}{(r-l) !} b_{n, l} \tau_{11}^{(m+n, r-l)} .
$$

For $r=1$ Eq. (2.36) becomes

$$
\left[\tau_{11}^{(m, 1)}, M_{11}^{(n)}\right]_{f}=b_{n, 1} M_{11}^{(m+n)}
$$

master-symmetries of degree 1 generate equations which belong to the given hierarchy.

\section{Lie and Poisson Brackets for Equations in $2+1$}

In this section we first derive an isomorphism between Lie and Poisson brackets. Then, using this isomorphism and the Lie algebra of the operators $\hat{K}_{12}^{0}$, we prove that $\Theta_{12}^{-1} \widehat{K}_{12}^{0} H_{12}$ are extended gradients. This implies that all extended symmetries of the previous section give rise to conserved quantities.

Theorem 3.1. Let $[a, b]_{L}=a_{L}[b]-b_{L}[a]$ be a Lie commutator and $\langle f, g\rangle$ be an appropriate symmetric bi-linear form. Let grad $I$ be the gradient of a functional $I$, defined by $I_{L}[v]=\langle\operatorname{grad} I, v\rangle$; then $\gamma$ is a gradient function iff $\gamma_{L}=\gamma_{L}^{*}$, where $M^{*}$ denotes the adjoint of the operator $M$ with respect to the above bi-linear form, i.e. $\left\langle M^{*} f, g\right\rangle=\langle f, M g\rangle$. Then if the operator $\Theta$ is a Hamiltonian operator, i.e. if

$$
\Theta^{*}=-\Theta,\left\langle a, \Theta_{L}[\Theta b] c\right\rangle+\text { cyclic permut }=0,
$$


it follows that

$$
[\Theta f, \Theta g]_{L}=\Theta \operatorname{grad}\langle f, \Theta g\rangle+\Theta\left\{\left(f_{L}-f_{L}^{*}\right)[\Theta g]-\left(g_{L}-g_{L}^{*}\right)[\Theta f]\right\} .
$$

Proof.

$$
\begin{aligned}
\operatorname{grad}\langle f, \Theta g\rangle[v] & =\left\langle f_{L}[v], \Theta g\right\rangle+\left\langle f, \Theta_{L}[v] g\right\rangle+\left\langle f, \Theta_{L}[v]\right\rangle \\
& =\left\langle f_{L}^{*}[\Theta g]-g_{L}^{*}[\Theta f]\right\rangle=\left\langle f_{L}^{*}[\Theta g]+M_{g}^{*} f-g_{L}^{*}[\Theta g], v\right\rangle,
\end{aligned}
$$

where $\left\langle f, \Theta_{L}[v] g\right\rangle=\left\langle f, M_{g}[v]\right\rangle$ and $M_{g}$ denotes a linear operator depending on g. Hence

$$
\begin{aligned}
& {[\Theta f, \Theta g]_{L}-\Theta \operatorname{grad}\langle f, \Theta g\rangle=\Theta_{L}[\Theta g] f+\Theta f_{L}[\Theta g]-\Theta_{L}[\Theta f] g-\Theta g_{L}[\Theta f]} \\
& \quad-\Theta f_{L}^{*}[\Theta g]+\Theta g_{L}^{*}[\Theta g]-\Theta M_{L}^{*} f \\
& =\Theta_{L}[\Theta g] f-\Theta_{L}[\Theta f] g-\Theta M_{g}^{*} f+\Theta\left\{\left(f_{L}-f_{L}^{*}\right)[\Theta g]-\left(g_{L}-g_{L}^{*}\right)[\Theta f]\right\} .
\end{aligned}
$$

But the sum of the first three terms of the above equals zero because of (3.1). Hence (3.2) follows.

In the above $a_{L}$ denotes an appropriate directional derivative. For equations in $1+1$ :

$$
[a, b]_{L}=[a, b]_{f},\langle f, g\rangle=\int_{\mathbb{R}} d x \text { trace } g f .
$$

For equations in $2+1$,

$$
\begin{aligned}
{\left[a_{12}, b_{12}\right]_{L}=} & {\left[a_{12}, b_{12}\right]_{d},\left\langle f_{11}, g_{11}\right\rangle=\int_{\mathbb{R}^{2}} d x d y \operatorname{trace} g_{11} f_{11}, } \\
& \cdot\left\langle f_{12}, g_{12}\right\rangle=\int_{\mathbb{R}^{3}} d x d y_{1} d y_{2} \text { trace } g_{21} f_{12}
\end{aligned}
$$

(if $f$ and $g$ are scalars, then delete trace), where $[,]_{f},[,]_{d}$ are defined in (2.13), (2.4). Furthermore the following double representation of the functional $I$

$$
I=\int_{\mathbb{R}^{2}} d x d y_{1} \text { trace } \rho_{11}=\int_{\mathbb{R}^{3}} d x d y_{1} d y_{2} \text { trace } \rho_{12}
$$

allows us to define the extended gradient $\operatorname{grad}_{12} I$ and the gradient $\operatorname{grad} I$ of the functional $I$ by

$$
\begin{aligned}
& I_{d}\left[v_{12}\right]=\int_{\mathbb{R}^{3}} d x d y_{1} d y_{2} \delta_{12} \operatorname{trace} \rho_{12}\left[v_{12}\right] \doteqdot\left\langle\operatorname{grad}_{12} I, v_{12}\right\rangle, \\
& I_{f}\left[v_{11}\right]=\int_{\mathbb{R}^{2}} d x d y_{1} \operatorname{trace} \rho_{11_{f}}\left[v_{11}\right] \doteqdot\left\langle\operatorname{grad} I, v_{11}\right\rangle .
\end{aligned}
$$

The following theorem, proven in [1], maps extended gradients $\gamma_{12}$ to gradients $\gamma_{11}$ :

\section{Theorem 3.2.}

a) $\gamma_{12}$ and $\gamma_{11}$ are extended gradients and gradients respectively iff $\gamma_{12_{d}}^{*}=\gamma_{12_{d}}$ and $\gamma_{11_{f}}^{*}=\gamma_{11_{f}}$, with respect to the bilinear forms (3.4c) and (3.4b) respectively.

b) If $\gamma_{12}$ is an extended gradient, then $\gamma_{11}$ is a gradient corresponding to the same potential, namely if $\gamma_{12}=\operatorname{grad}_{12} I$, then $\gamma_{11}=\operatorname{grad} I$. 
Proposition 3.1. Assume that the hereditary operator $\Phi_{12}$ is a strong symmetry for the starting symmetries $\hat{M}_{12} H_{12}$ and $\hat{N}_{12} H_{12}$. Further assume that $\hat{M}_{12}, \hat{N}_{12}$ form a Lie algebra (analogous to (1.7) and (1.13)) and that $\Theta_{12}$ is a Hamiltonian operator whose inverse exists. Then

$$
\Theta_{12}^{-1} \Phi_{12}^{m} \hat{K}_{12}^{0} H_{12}, \quad \hat{K}_{12}^{0}=\hat{M}_{12} \quad \text { or } \quad \hat{N}_{12}
$$

are extended gradients, proved that $\Theta_{12}^{-1} \hat{K}_{12}^{0} H_{12}$ are extended gradients.

Proof. For concreteness we proof the above proposition for the recursion operator and starting symmetries associated with the two dimensional Schrödinger and $2 \times 2$ AKNS problems.

\section{III.A. Conserved Quantities for Equations Related to KP Equation}

Corollary 3.1. Let

$$
\begin{aligned}
& \hat{N}_{12} \doteqdot q_{12}^{-}, \quad \hat{M}_{12} \doteqdot D q_{12}^{+}+q_{12}^{-} D^{-1} q_{12}^{-}, \quad H_{12} \doteqdot H\left(y_{1}, y_{2}\right), \\
& \hat{M}_{12}^{(n)} \doteqdot \Phi_{12}^{n} \hat{M}_{12}, \quad \hat{N}_{12}^{(n)}=\Phi_{12}^{n} \hat{N}_{12}, \quad \Theta_{12}=D,
\end{aligned}
$$

where $\Phi_{12}$ is the recursion operator associated with the KP and is defined by (1.4). Then

$$
\begin{aligned}
D^{-1} \hat{M}^{(n+1)} H_{12}^{(3)} & =\operatorname{grad}\left\langle\hat{M}^{(n)} H_{12}^{(1)}, D^{-1} \hat{N}^{(1)} H_{12}^{(2)}\right\rangle, \\
D^{-1} \hat{N}^{(n+1)} H_{12}^{(3)} & =\operatorname{grad}\left\langle\hat{M}^{(n)} H_{12}^{(1)}, D^{-1} \hat{M} H_{12}^{(2)}\right\rangle .
\end{aligned}
$$

Proof. We first note that the assumptions of Proposition 3.1 are fulfilled. Namely $\Phi_{12}$ is hereditary and is a strong symmetry of $\hat{M}_{12} H_{12}, \hat{N} H_{12}$, (see Lemma 4.2 and Appendix C.1a of [1]). The operator $D^{-1}$ is obviously a Hamiltonian operator. Furthermore, $D^{-1} \widehat{M}_{12} H_{12}$ is an extended gradient (see Appendix A). Since $D^{-1} \hat{M}_{12} H_{12}$ is an extended gradient, Theorem 3.1 and $(1.7 \mathrm{c})$ imply that $D^{-1} \hat{N}_{12}^{(1)} H_{12}$ is an extended gradient. Then Theorem 3.1 and $\left[\hat{M}^{(n)} H_{12}^{(1)}\right.$, $\left.\hat{N}^{(1)} H_{12}^{(2)}\right]_{d}=-\hat{M}^{(n+1)} H_{12}^{(3)}$ imply by induction (3.9a). Finally Theorem 3.1 and $\left[\hat{M}^{(n)} H_{12}^{(1)}, \hat{M} H_{12}^{(2)}\right]_{d}=-\hat{N}^{(n+1)} H_{12}^{(3)}$ imply by induction $(3.9 \mathrm{~b})$.

A consequence of the above result is that all symmetries derived in Sect. II.B. give rise to conserved quantities. For example, the following $t$-dependent extended symmetries (see $(2.19 b)$ and $(2.21 \mathrm{a}))$

$$
\begin{aligned}
& \sigma_{12}^{(1)}=\hat{M}_{12}^{(m)}\left(y_{1}+y_{2}\right)+t 12 \alpha \hat{N}_{12}^{(m+1)} \cdot 1, \\
& \sigma_{12}^{(2)}=\hat{N}_{12}^{(m)}\left(y_{1}+y_{2}\right)^{2}+t 24 \alpha \hat{M}_{12}^{(m)} \cdot\left(y_{1}+y_{2}\right)+t^{2} 144 \alpha^{2} \hat{N}_{12}^{(m+1)} \cdot 1,
\end{aligned}
$$

of the KP equation $q_{1_{t}}=M_{11}^{(1)}=2\left(q_{1_{x x x}}+6 q_{1} q_{1_{x}}+3 \alpha^{2} D^{-1} q_{1_{y_{1} y_{1}}}\right)$ correspond to extended gradient functions $D^{-1} \sigma_{12}^{(1)}$ and $D^{-1} \sigma_{12}^{(2)}$; then they give rise to the following $t$-dependent conserved quantities (see Eqs. (4.15))

$$
\begin{aligned}
& I^{(1)}=\int_{\mathbb{R}^{2}} d x d y_{1}\left(\frac{1}{2(2 m+3)}\left(D^{-1} \hat{M}_{12}^{(m+1)}\left(y_{1}+y_{2}\right)\right)_{11}+\frac{3 \alpha t}{m+2}\left(D^{-1} \hat{N}_{12}^{(m+2)} \cdot 1\right)_{11}\right), \\
& I^{(2)}=\int_{\mathbb{R}^{2}} d x d y_{1}\left(\frac{1}{4(m+1)}\left(D^{-1} \hat{N}^{(m+1)}\left(y_{1}+y_{2}\right)^{2}\right)_{11}\right.
\end{aligned}
$$




$$
\left.+\frac{t 12 \alpha}{2 m+3}\left(D^{-1} \hat{M}_{12}^{(m+1)}\left(y_{1}+y_{2}\right)\right)_{11}+\frac{t^{2} 36 \alpha^{2}}{m+2}\left(D^{-1} \hat{N}_{12}^{(m+2)} \cdot 1\right)_{11}\right) .
$$

\section{III.B. Conserved Quantities for Equations Related to DS Equation}

Corollary 3.2. Let

$$
\hat{M}_{12} \doteqdot Q_{12} \sigma, \quad \hat{N}_{12} \doteqdot Q_{12}^{-},
$$

$H_{12}$ diagonal and such that

$$
P_{12} H_{12}=0, \quad \hat{M}_{12}^{(n)} \doteqdot \Phi_{12}^{n} \hat{M}_{12}, \quad \hat{N}_{12}^{(n)}=\Phi_{12}^{n} \hat{N}_{12}, \quad \Theta_{12}=\sigma,
$$

where $\Phi_{12}$ is the recursion operator associated with the DS equation and is defined by (1.9). Then

$$
\begin{aligned}
\sigma \hat{M}^{(n+1)} H_{12}^{(3)} & =\operatorname{grad}\left\langle\hat{M}^{(n)} H_{12}^{(1)}, \sigma \hat{N}^{(1)} H_{12}^{(2)}\right\rangle, \\
\sigma \hat{N}^{(n)} H_{12}^{(3)} & =\operatorname{grad}\left\langle\hat{M}^{(n)} H_{12}^{(1)}, \sigma \hat{M} H_{12}^{(1)}\right\rangle .
\end{aligned}
$$

Proof. The assumptions of Proposition 3.1 are again fulfilled (see Lemma 4.2 and Appendix C.2a of [1]). The operator $\sigma$ is obviously Hamiltonian in a space of off-diagonal matrices. Furthermore, $\sigma \hat{M}_{12} H_{12}, \sigma \phi_{12} \widehat{N}_{12} H_{12}$ are extended gradients (see Appendix A).

Since the above are gradients, $\left[\hat{M}^{(n)} H_{12}^{(1)}, \hat{N}^{(1)} H_{12}^{(2)}\right]_{d}=-\hat{M}^{(n+1)} H_{12}^{(3)}$ implies (3.11a). Then $\left[\hat{M}^{(n)} H_{12}^{(1)}, \hat{M} H_{12}^{(2)}\right]=-\hat{N}^{(n)} H_{12}^{(3)}$ implies (3.11b).

The above implies that the symmetries derived in Sect. II.C. give rise to conserved quantities. For example, the $1^{\text {st }}$ and $2^{\text {nd }}$ order $t$-dependent symmetries

$$
\begin{aligned}
& \sigma_{12}^{(1)}=\hat{M}_{12}^{(m)} H_{12}^{(1)}-8 \alpha t \hat{N}_{12}^{(m)} \cdot I, \\
& \sigma_{12}^{(2)}=\hat{N}_{12}^{(m)} H_{12}^{(2)}-t 16 \alpha \hat{M}_{12}^{(m)} H_{12}^{(1)}+t^{2} 64 \alpha^{2} \hat{N}_{12}^{(m+2)} \cdot I,
\end{aligned}
$$

of the DS equation $Q_{1_{t}}=M_{11}^{(2)}=-\left[2 \sigma\left(Q_{I_{x x}}+\alpha^{2} Q_{1_{y_{1} y_{1}}}\right)-Q_{1} A_{1}+A_{1} Q_{1}\right]$, $\left(D-J D_{1}\right) A_{1}=-2\left(D+J D_{1}\right) \sigma Q_{1}^{2}$, obtained from Eqs. (2.29-30), correspond to the extended gradients $\sigma \sigma_{12}^{(1)}, \sigma \sigma_{12}^{(2)}$; then they give rise to the following $t$-dependent conserved quantities (see Eqs. (4.24)):

$$
\begin{aligned}
I^{(1)}= & \int_{\mathbb{R}^{2}} d x d y \operatorname{trace} \sigma\left[Q_{1}, \frac{1}{2(m+1)}\left(D^{-1} \hat{M}_{12}^{(m+1)} H_{12}^{(1)}\right)_{11}-\frac{t 4 \alpha}{m+1} \hat{N}_{12}^{(m+1)} \cdot I\right], \\
I^{(2)=} & \int_{\mathbb{R}^{2}} d x d y_{1} \operatorname{trace} \sigma\left[Q_{1}, \frac{1}{2(m+1)}\left(D^{-1} \hat{N}_{12}^{(m+1)} H_{12}^{(2)}\right)_{11}\right. \\
& \left.-\frac{t 8 \alpha}{m+1}\left(D^{-1} \hat{M}_{12}^{(m+1)} H_{12}^{(1)}\right)_{11}+\frac{t^{2} 32 \alpha^{2}}{m+3}\left(D^{-1} \hat{N}_{12}^{(m+3)} \cdot I\right)_{11}\right] .
\end{aligned}
$$

\section{On a Non-Gradient Master-Symmetry}

In this section we make extensive use of the isomorphism between Lie and Poisson brackets. Hence it is useful to investigate the properties of

$$
\Theta\left(g_{L}-g_{L}^{*}\right)=T_{L}+\Theta T_{L}^{*} \Theta^{-1} ; \quad T \doteqdot \Theta g, \quad \Theta_{L}=0 .
$$


Lemma 4.1. Let

$$
S \doteqdot \Phi_{L}[T]+\left[\Phi, T_{L}\right]
$$

with its adjoint

$$
S^{*}=\Phi_{L}^{*}[T]+\left[T_{L}^{*}, \Phi^{*}\right] .
$$

a) If $\Phi$ is hereditary then

$$
\Phi_{L}^{*}\left[\Phi^{n} T\right]+\left[\Phi^{n} T\right]_{L}^{*} \Phi^{*}-\Phi^{*}\left(\Phi^{n} T\right)_{L}^{*}=S^{*} \Phi^{*^{n}} .
$$

b) If $\Phi$ is factorizable in terms of compatible Hamiltonian operators, i.e. if $\Phi=\Omega \Theta^{-1}$, where $\Omega+v \Theta$ is a Hamiltonian operator, $\Theta$ is invertible and $v$ is an arbitrary constant, then

$$
(\Phi T)_{L}+\Theta(\Phi T)_{L}^{*} \Theta^{-1}=\Phi\left(T_{L}+\Theta T_{L}^{*} \Theta^{-1}\right)+\Theta S^{*} \Theta^{-1}
$$

where we have assumed for simplicity that $\Theta_{L}=0$.

c)

$$
\left(\Phi^{n} T\right)_{L}+\Theta\left(\Phi^{n} T\right)_{L}^{*} \Theta^{-1}=\Phi^{n}\left(T_{L}+\Theta T_{L}^{*} \Theta^{-1}\right)+\sum_{r=1}^{n} \Phi^{r-1} \Theta \Phi^{*^{n-r}} S^{*} \Theta^{-1}
$$

Proof. Equation (4.4) is the adjoint of (2.7) for $K=T$. Equation (4.5) is derived in Appendix B, and (4.6) follows from (4.5) by induction.

Theorem 4.1. Assume that $\Phi$ is factorizable in terms of compatible Hamiltonian operators and that $\Theta_{L}=0$. Further assume that $\Theta^{-1} \Phi^{n} M$ is a gradient function and that $\Phi$ is a strong symmetry for $M$. Then

$$
\begin{aligned}
\Phi^{m} \sum_{r=1}^{n} \Phi^{n-r} S \Phi^{r-1} M= & \Theta \operatorname{grad}\left\langle\Theta^{-1} \Phi^{n} M, \Phi^{m} T\right\rangle \\
& -\sum_{r=1}^{m} \Phi^{r-1} \Theta \Phi^{* m-r} S^{*} \Theta^{-1} \Phi^{n} M \\
& -\Phi^{m}\left(T_{L}+\Theta T_{L}^{*} \Theta^{-1}\right) \Phi^{n} M-\Phi^{n+m}[M, T]_{L} .
\end{aligned}
$$

Proof. Using the fact that $\Theta^{-1} \Phi^{n} M$ is a gradient, Eq. (3.2) becomes

$$
\left[\Phi^{n} M, \Phi^{m} T\right]_{L}=\Theta \operatorname{grad}\left\langle\Theta^{-1} \Phi^{n} M, \Phi^{m} T\right\rangle-\left\{\left(\Phi^{m} T\right)_{L}+\Theta\left(\Phi^{m} T\right)_{L}^{*} \Theta^{-1}\right\} \Phi^{n} M .
$$

Since $M$ is a strong symmetry of $\Phi$, Theorem 2.1 implies

$$
\left[\Phi^{n} M, \Phi^{m} T\right]_{L}=\Phi^{n+m}[M, T]_{L}+\Phi^{m}\left(\sum_{r=1}^{n} \Phi^{n-r} S \Phi^{r-1}\right) M
$$

Using the above and (4.6) in (4.8) we obtain (4.7).

Equations (4.6) and (4.9) are useful in finding non-gradient master-symmetries for equations in $2+1$. Furthermore, Theorem 4.1 is useful for deriving the potentials of various gradients. Formulae (4.6), (4.9) and (4.7) take a particularly simple form 
if the function $T_{12}$ is such that

i)

$$
S_{12}=S_{12}^{*}=c 1,
$$

where 1 is the identity operator and $c$ is an arbitrary constant, and

ii)

$$
T_{12_{d}}+\Theta_{12} T_{12_{d}}^{*} \Theta_{12}^{-1}=0 .
$$

In the following two examples the non-gradient master-symmetries are generated through functions $T_{12}$ that satisfy Eqs. (4.10).

\section{IVA. Equations Associated with the KP Equation}

\section{Corollary 4.1.}

a) $\Phi_{12}^{2} \delta_{12}$ is a non-gradient master-symmetry for the KP and the equations related to $\mathrm{KP}$ :

$$
\begin{gathered}
{\left[\Phi_{12}^{n} \hat{K}_{12}^{0} H_{12}, \Phi_{12}^{2} \delta_{12}\right]_{d}=b_{n} \Phi_{12}^{n+1} \hat{K}_{12}^{0} H_{12}} \\
8 \Phi_{12}=\left(\Phi_{12}^{2} \delta_{12}\right)_{d}+\Theta_{12}\left(\Phi_{12}^{2} \delta_{12}\right)_{d}^{*} \Theta_{12}^{-1}
\end{gathered}
$$

where $b_{n}$ and $H_{12}$ are given by

$$
b_{n}=4 n, \quad H_{12}=H\left(y_{1}, y_{2}\right) \text { arbitrary, if } \hat{K}_{12}^{0}=\hat{N}_{12},
$$

and by

$$
b_{n}=2(2 n+1), \quad H_{12}=\left(y_{1}+y_{2}\right)^{r}, \quad r=0,1, \quad \text { if } \hat{K}_{12}^{0}=\hat{M}_{12} .
$$

b) Let

Then

$$
\hat{\gamma}_{12}^{(n)} \doteqdot \Phi_{12}^{*^{n}} \hat{\gamma}_{12}^{0}, \quad \hat{\gamma}_{12}^{0} \doteqdot \Theta_{12}^{-1} \hat{K}_{12}^{0}
$$

$$
\begin{aligned}
I_{n} & \doteqdot \frac{1}{b_{n+1}}\left\langle\hat{\gamma}_{12}^{(n+1)} H_{12}, \delta_{12}\right\rangle=\frac{1}{b_{n+1}}-\int_{\mathbb{R}^{3}} d x d y_{1} d y_{2} \delta_{12} \hat{\gamma}_{12}^{(n+1)} H_{12} \\
& =\frac{1}{b_{n+1}-} \int_{\mathbb{R}^{2}} d x d y_{1}\left(\hat{\gamma}_{12}^{(n+1)} H_{12}\right)_{11},
\end{aligned}
$$

where $b_{n}$ and $H_{12}$ are given in (4.13).

Proof. If

$$
T_{12}=\delta_{12},
$$

Eq. (4.10b) is trivially satisfied and Eq. (4.10a) holds for $c=4$, since $\Phi_{12_{d}}\left[\delta_{12}\right]=$ $\Phi_{12 d}^{*}\left[\delta_{12}\right]=4$. Equation (4.12) is a simple consequence of (4.6) for $n=2$; using the following results

$$
\begin{gathered}
\Phi_{12}^{n}\left[\hat{N}_{12} H_{12}, \delta_{12}\right]_{d}=0, \\
\Phi_{12}^{n}\left[\hat{M}_{12}\left(y_{1}+y_{2}\right)^{r}, \delta_{12}\right]_{d}=2 \Phi_{12}^{n-1} \hat{M}_{12}\left(y_{1}+y_{2}\right)^{r}, \quad r=0,1,
\end{gathered}
$$

(see Appendix A) in Eqs. (4.9) and (4.7) (with $M=\hat{K}_{12}^{0} H_{12}$ and $H_{12}$ as in (4.13)), we obtain

$$
\left[\Phi_{12}^{n} \hat{K}_{12}^{0} H_{12}, \Phi_{12}^{m} \delta_{12}\right]_{d}=b_{n} \Phi_{12}^{n+m-1} \hat{K}_{12}^{0} H_{12}
$$


(that reduces to (4.11) for $m=2$ ), and

$$
b_{n} \Phi_{12}^{n+m-1} \hat{K}_{12}^{0} H_{12}=\Theta_{12} \operatorname{grad}_{12}\left\langle\hat{\gamma}_{12}^{(n)} H_{12}, \Phi_{12}^{m} \delta_{12}\right\rangle \text {, }
$$

where we have used $\Phi_{12}^{n} \Theta_{12}=\Theta_{12} \Phi_{12}^{*^{n}}$. Equation (4.19) reduces to (4.15) if one uses the definition of $\left\langle f_{12}, g_{12}\right\rangle$ given by (1.20) and (3.4c).

Remark 4.1.

i) $T \doteqdot \Phi^{2} 1$ is a non-gradient master-symmetry for the $\mathrm{KdV}$ equation. Given $T$ one recovers $\Phi$ from $T_{f}+\Theta T_{f}^{*} \Theta^{-1}$. Equation (4.12) is the two-dimensional analogue of this well known formula [8]-[10].

ii) Theorem 3.2 implies that Eqs. (4.15) with $m=1, H_{12}=1$ reduce to the following formula [6]:

$$
\gamma_{11}^{(n)}=\frac{1}{b_{n+1}} \operatorname{grad} \int_{\mathbb{R}^{2}} d x d y_{1} \gamma_{11}^{(n+1)}
$$

An analogous formula, for the $\mathrm{KdV}$ equation is well known

$$
\gamma^{(n)}=\frac{1}{2(2 n+3)} \operatorname{grad} \int_{\mathbb{R}} d x \gamma^{(n+1)} .
$$

iii) We observe that Eq. (4.18) for $H_{12}=1$ cannot be projected into Eq. (2.37).

IVB. Equations Associated with the DS Equation

\section{Corollary 4.2.}

a) $\Phi_{12}^{2} T_{12}, T_{12} \doteqdot(x / 2) \sigma Q_{12}^{+} \delta_{12} I, I=\operatorname{diag}(1,1)$, is a non-gradient master-symmetry for the DS and the equations related to DS:

$$
\begin{gathered}
{\left[\Phi_{12}^{n} \hat{K}_{12}^{0} H_{12}, \Phi_{12}^{2} T_{12}\right]_{d}=n \Phi_{12}^{n+1} \hat{K}_{12}^{0} H_{12},} \\
2 \Phi_{12}=\left(\Phi_{12}^{2} T_{12}\right)_{d}+\Theta_{12}\left(\Phi_{12}^{2} T_{12}\right)_{d}^{*} \Theta_{12}^{-1}, \quad \Theta_{12}=\sigma,
\end{gathered}
$$

where $\hat{K}_{12}^{0} H_{12}$ is defined in $(1.11-12)$.

b) Let

$$
\hat{\gamma}_{12}^{(n)}=\Phi_{12}^{*_{12}^{n}} \hat{\gamma}_{12}^{0}, \quad \hat{\gamma}_{12}^{0}=\Theta_{12}^{-1} \hat{K}_{12}^{0}, \quad \Theta_{12}=\sigma
$$

Then

$$
\begin{gathered}
\hat{\gamma}_{12}^{(n)} H_{12}=\operatorname{grad}_{12} I_{n}, \\
I_{n} \doteqdot \frac{1}{n+1}\left\langle\hat{\gamma}_{12}^{(n+1)} H_{12}, T_{12}\right\rangle=-\frac{1}{2(n+1)} \int_{\mathbb{B}^{3}} d x d y_{1} d y_{2} \operatorname{trace} \delta_{12} Q_{12}^{+} \sigma \hat{\gamma}_{12}^{(n+1)} H_{12} \\
=\frac{1}{2(n+1)} \int_{\mathbb{R}^{2}} d x d y_{1} \operatorname{trace} \sigma\left[Q_{1},\left(\tilde{\gamma}_{12}^{(n+1)} H_{12}\right)_{11}\right] .
\end{gathered}
$$

Proof. If

$$
T_{12} \doteqdot \frac{x}{2} \sigma Q_{12}^{+} \delta_{12} I
$$

Eq. (4.10b) is satisfied and Eq. (4.10a) holds for $c=1$ (see Appendix A). Then the 
derivation of Eqs. (4.21), (4.22) and (4.24) is analogous to the one of Corollary 4.1. (see Appendix A).

\section{2+ 1 Dimensional Equations as Reductions of Non-Local Systems}

In [1] and [14] the classes of evolution equations

$$
q_{1 t}=\int_{\mathbb{R}} d y_{2} \delta_{12} \Phi_{12}^{n} \hat{K}_{12}^{0} \cdot 1,
$$

where $\Phi_{12}$ and $\hat{K}_{12}^{0}$ are defined in (1.4-5), were algorithmically derived from the spectral problem

$$
w_{x x}+q(x, y) w+\alpha w_{y}=0 .
$$

In this section we show that Eqs. (5.1) are exact reductions of equations non-local in $y$, generated by the following non-local analogue of (5.2):

where

$$
w_{x x}+\tilde{q} w+\alpha w_{v}=\lambda w
$$

$$
(\tilde{q} f)(x, y) \doteqdot \int_{\mathbb{R}} d y_{2} q\left(x, y, y_{2}\right) f\left(x, y_{2}\right) \text {. }
$$

Hei'eafter the symbols $\tilde{u}$ and $u_{12}$ indicate the integral operator defined by

$$
(\tilde{u} f)(x, y) \doteqdot \int_{\mathbb{R}} d \dot{y}_{2} u\left(x, y, y_{2}\right) f\left(x, y_{2}\right)
$$

and its kernel $u_{12} \doteqdot u\left(x, y_{1}, y_{2}\right)$, respectively.

The algorithmic derivation of the classes of evolution equations associated with (5.3) is standard; its main steps are:

i) Compatibility. A compatibility between Eq. (5.3), written in the more convenient form $\left(\begin{array}{l}w \\ w_{x}\end{array}\right)_{x}=\left(\begin{array}{cc}0, & 1 \\ \lambda-\tilde{q}-D_{y}, & 0\end{array}\right)\left(\begin{array}{l}w \\ w_{x}\end{array}\right)$, and the linear evolution equation $\left(\begin{array}{c}w \\ w_{x}\end{array}\right)_{t}=\tilde{V}\left(\begin{array}{l}w \\ w_{x}\end{array}\right)$, yields the following operator equation:

$$
\begin{aligned}
\tilde{q}_{t}= & \tilde{c}_{x x x}+\left[\tilde{q}+\alpha D_{y}, \tilde{c}\right]_{x}^{+}+\left[\tilde{q}+\alpha D_{y}, \tilde{c}_{x}\right]^{+}+\left[\tilde{q}+\alpha D_{y}, D^{-1}\left[\tilde{q}+\alpha D_{y}, \tilde{c}\right]\right] \\
& -4 \lambda \tilde{c}_{x}+\tilde{A}_{0}\left(\tilde{q}+\alpha D_{y}\right)-\left(\tilde{q}+\alpha D_{y}\right) \tilde{A}_{0},
\end{aligned}
$$

where the scalar integral operator $2 \tilde{c}$ is the 1,2 component of the $2 \times 2$ matrix integral operator $\tilde{V}, A_{0_{x}}=0$ and $[$,$] and [,]^{+}$are the usual commutator and anticommutator.

ii) Equation for the kernel. The operator equation (5.6), together with the definition (5.5), implies the following equation for the kernels $q_{12}, c_{12}, A_{12}$ :

where

$$
q_{12_{t}}=D \tilde{\Psi}_{12} C_{12}-\tilde{q}_{12}^{-} A_{12}+-4 \lambda c_{12_{x}},
$$

$$
\begin{gathered}
\tilde{\Psi}_{12} \doteqdot D^{2}+\tilde{q}_{12}^{+}+D^{-1} \tilde{q}_{12}^{+} D+D^{-1} \tilde{q}_{12}^{-} D^{-1} \tilde{q}_{12}^{-}, \\
\tilde{q}_{12}^{ \pm} f_{12}=\int_{\mathbb{R}}\left(q_{13} f_{32} \pm f_{13} g_{32}\right) d y_{3}+\alpha\left(D_{1} \mp D_{2}\right) f_{12} .
\end{gathered}
$$

iii) Expansion in powers of $\lambda$. Let us first assume that

$$
C_{12}=\sum_{j=0}^{n} \lambda^{j} C_{12}^{(j)}, \quad A_{12}=0,
$$


equating the coefficients of $\lambda^{j}(0 \leqq j \leqq n)$ to zero we obtain: $C_{12}^{(n)}=H_{12}^{(n)} ; C_{12}^{(j-1)}=$ $\frac{1}{4} \widetilde{\Psi}_{12} C_{12}^{(j)}+H_{12}^{(j-1)}(1 \leqq j \leqq n) ; \quad q_{12}=D \widetilde{\Psi}_{12} C_{12}^{(0)}$; where $H_{12}^{(j)}=H^{(j)}\left(y_{1}, y_{2}\right)$. Then $C_{12}^{(0)}=\sum_{s=0}^{n} 4^{s-n} \tilde{\Psi}_{12}^{n-s} H_{12}^{(n-s)}$ and

$$
q_{12 t}=\sum_{s=0}^{n} 4^{s-n} D \tilde{\Psi}_{12}^{n-s+1} H_{12}^{(n-s)}=\sum_{s=0}^{n} 4^{s-n} \tilde{\Phi}_{12}^{n-s+1} \cdot H_{12}^{(n-s)}
$$

where

$$
\tilde{\Phi}_{12} \doteqdot D \widetilde{\Psi}_{12} D^{-1}=D^{2}+\tilde{q}_{12}^{+}+D \tilde{q}_{12}^{+} D^{-1}+\tilde{q}_{12}^{-} D^{-1} \tilde{q}_{12}^{-} D^{-1}
$$

If we assume that

$$
C_{12}=\sum_{j=0}^{n} \lambda_{i}^{j} C_{12}^{(j)}, \quad A_{12}=-4 \sum_{j=0}^{n+1} \lambda^{j} \bar{H}_{12}^{(j)}, \quad \bar{H}_{12}^{(j)}=\bar{H}^{(j)}\left(y_{1}, y_{2}\right)
$$

then $C_{12}^{(n)}=D^{-1} \tilde{q}_{12}^{-} \cdot \bar{H}_{12}^{(n+1)}+H_{12}^{(n)} ; C_{12}^{(j-1)}=\frac{1}{4} \tilde{\Psi}_{12} C_{12}^{(j)}+D^{-1} \tilde{q}_{12} \cdot H_{12}^{(j)}+H_{12}^{(j-1)}(1 \leqq$ $j \leqq n) ; q_{12}=D \tilde{\Psi}_{12} C_{12}^{(0)}+4 \tilde{q}_{12}^{-} \bar{H}_{12}^{(0)}$, where $H_{12}^{(j)}=H^{(j)}\left(y_{1}, y_{2}\right)$. The choice $H_{12}^{(j)}=0$ for $0 \leqq j \leqq n$ yields $C_{12}^{(0)}=\sum_{s=0}^{n} 4^{s-n} \tilde{\Psi}_{12}^{n-s} D^{-1} \tilde{q}_{12}^{-} \cdot \bar{H}_{12}^{(n-s+1)}$ and

$$
q_{12 t}=\sum_{s=0}^{n+1} 4^{s-n} D \tilde{\Psi}_{12}^{n-s+1} D^{-1} \tilde{q}_{12}^{-} \cdot \bar{H}_{12}^{(n-s+1)}=\sum_{s=0}^{n+1} 4^{s-n} \tilde{\Phi}_{12}^{n-s+1} \tilde{q}_{12}^{-} \cdot \bar{H}_{12}^{(n-s+1)}
$$

Thus the isospectral problem (5.3) generates the classes of evolution equations (5.10) and (5.12).

It turns out that the transformation $q_{12} \rightarrow \delta_{12} q_{1}, q_{1}=q\left(x, y_{1}\right)$, is an exact reduction of Eqs. $(5.10-11)$ if, at the same time, $4^{s-n} H_{12}^{(n-s)}, 4^{s-n} \bar{H}_{12}^{(n+1-s)} \rightarrow$ $\beta^{s}\left(\begin{array}{c}n+1 \\ s\end{array}\right) \delta_{12}^{s}$. In this case $\tilde{q}_{12}^{ \pm} \rightarrow q_{12}^{ \pm}, \tilde{\Phi}_{12} \rightarrow \Phi_{12}$ and

$$
\begin{gathered}
\delta_{12} q_{1_{t}}=\sum_{l=0}^{n+1} \beta^{l}\left(\begin{array}{c}
n+1 \\
l
\end{array}\right) \Phi_{12}^{n+1-l} D \cdot \delta_{12}^{l}=\delta_{12} \Phi_{12}^{n+1} D \cdot 1=\delta_{12} \Phi_{12}^{n} \hat{M}_{12} \cdot 1, \\
\delta_{12} q_{1 t}=\sum_{l=0}^{n+1} \beta^{l}\left(\begin{array}{c}
n+1 \\
l
\end{array}\right) \Phi_{12}^{n-l+1} q_{12}^{-} \cdot \delta_{12}^{l}=\delta_{12} \Phi_{12}^{n} q_{12}^{-} \cdot 1=\delta_{12} \Phi_{12}^{n} \hat{N}_{12} \cdot 1 .
\end{gathered}
$$

Proceeding exactly in the same way it is possible to show that the nonlocal eigenvalue problem

$$
W_{x}=J W_{y}+\tilde{Q} W+\lambda J W
$$

generates the following class of evolution equations:

$$
Q_{12_{1}}=\sum_{l=0}^{n} a_{n, l} \tilde{\Phi}_{12}^{n-l} \tilde{Q}_{12}^{-} \cdot H_{12}^{(l)}, \quad Q_{12} \doteqdot Q\left(x, y_{1}, y_{2}\right)
$$

where

$$
\begin{gathered}
\tilde{\Phi}_{12} F_{12} \doteqdot \sigma\left(P_{12}-\tilde{Q}_{12}^{+} P_{12}^{-1} \tilde{Q}_{12}^{+}\right) F_{12}, \quad F_{12} \doteqdot F\left(x, y_{1}, y_{2}\right) \text { off-diagonal } \\
\tilde{Q}_{12}^{+} F_{12} \doteqdot \int_{\mathbb{B}} d y_{3}\left(Q_{13} F_{32} \pm F_{13} Q_{32}\right)
\end{gathered}
$$


$\sigma=\operatorname{diag}(1,-1)$ and $H_{12}^{(j)}$ is defined by

$$
P_{12} H_{12}^{(j)}=0, \quad H_{12}^{(j)} \text { diagonal. }
$$

Also in this case the transformation $Q_{12} \rightarrow \delta_{12} Q_{1}$ is a reduction of (5.15) if $a_{n, l} \rightarrow \beta^{l}\left(\begin{array}{l}n \\ l\end{array}\right)(\beta=2 \alpha)$ and $H_{12}^{(l)} \rightarrow \delta_{12}^{l} I$ or $\delta_{12}^{l} \sigma$. In fact, $\tilde{Q}_{12}^{ \pm} \rightarrow Q_{12}^{ \pm}, \tilde{\Phi}_{12} \rightarrow \Phi_{12}$. Thus one obtains the following classes of equations:

$$
\delta_{12} Q_{1 t}=\sum_{l=0}^{n} \beta^{l}\left(\begin{array}{l}
n \\
l
\end{array}\right) \Phi_{12}^{n-l} Q_{12}^{-} \delta_{12}^{l} I=\delta_{12} \Phi_{12}^{n} Q_{12}^{-} I
$$

or

$$
\delta_{12} Q_{1_{t}}=\sum_{l=0}^{n} \beta^{l}\left(\begin{array}{l}
n \\
l
\end{array}\right) \Phi_{12} Q_{12}^{-} \delta_{12}^{l} \sigma=\delta_{12} \Phi_{12}^{n} Q_{12}^{-} \sigma
$$

associated with the eigenvalue problem

$$
W_{x}=J W_{y}+W Q+\lambda J W
$$

The above results clearly imply that all the notions introduced in [1] to characterize the algebraic properties of equations in $2+1$ dimensions can be justified and interpreted in terms of the algebraic structure of the corresponding non-local versions. For example:

i) The above derivations both motivate and explain the derivation of the recursion operators introduced in [1] and [14]. In particular the crucial role played by the integral representation of differential operators is clarified.

ii) The directional derivative introduced in [1], which is the main tool needed to investigate the algebraic properties of equations in $2+1$ dimensions, can be derived from the usual Frechét derivative in the space of non-local operators. For example, the Frechét derivative of $\tilde{q}_{12}^{ \pm} g_{12}$ in a direction $f_{12}$ is

$$
\begin{gathered}
\tilde{q}_{12}^{ \pm \prime}\left[f_{12}\right] g_{12}=f_{12}^{ \pm} g_{12}, \\
f_{12}^{ \pm} g_{12} \doteqdot \int_{\mathbb{R}} d y_{3}\left(f_{13} g_{32} \pm g_{13} f_{32}\right)
\end{gathered}
$$

which is exactly the direction derivative $q_{12_{d}}^{ \pm}\left[f_{12}\right] g_{12}$ introduced in [1].

iii) The definition of an admissible function and of its derivative follows from the fact that reduced functions admit a double representation; for example (5.13b) implies

$$
\sum_{l=0}^{n} \beta^{l}\left(\begin{array}{l}
n \\
l
\end{array}\right) \Phi_{12}^{n-l} q_{12}^{-} \delta_{12}^{l}=\delta_{12} \Phi_{12}^{n} q_{12}^{-} \cdot 1
$$

But the directional derivative is defined only on the admissible representation given by the left-hand side of (5.19), which is the form of the function before the reduction:

$$
\sum_{l=0}^{n} a_{n, l} \tilde{\Phi}_{12}^{n-l} \tilde{q}_{12}^{-} H_{12}^{(l)}
$$

In Appendix A we investigate (Eqs. (A.3)) the algebra of the nonlocal operators $a_{12}^{ \pm}$defined in (5.18b). Here we remark that this algebra can also be interpreted as an algebra of matrices in which \pm indicates the operations of anticommutator 
and commutater respectively, namely $a^{ \pm} b=a b \pm b a$. (See also Appendix C of [1].) This is not a coincidence and the following important observations, here illustrated on the recursion operator $\Phi_{12}$ of the KP class, can be made.

i) Integral operators:

$$
\begin{aligned}
q_{12}^{ \pm} f_{12} & =\int_{\mathbb{R}} d y_{3}\left(q_{13} f_{32} \pm f_{13} q_{32}\right), \\
q_{12} & =\delta_{12} q_{1}+\alpha \delta_{12}^{\prime},
\end{aligned}
$$

is equivalent to the introduction of the integral operator $\tilde{q}_{12}^{ \pm}$. Then $\Phi_{12}$ becomes the nonlocal recursion operator $\widetilde{\Phi}_{12}$, defined in (5.11) and associated with the nonlocal eigenvalue problem (5.3).

ii) Matrix operators:

$$
q^{ \pm} f \doteqdot q f \pm f q ; \quad q, f \text { matrices, }
$$

reduces $\Phi_{12}$ to the well-known matrix recursion operator

$$
\Phi \doteqdot D^{2}+q^{+}+D q^{+} D^{-1}+q^{-} D^{-1} q^{-} D^{-1},
$$

associated with the $N \times N$ matrix Schroedinger eigenvalue problem in one dimension [15].

The directional derivative $q_{12_{d}}^{ \pm}\left[f_{12}\right] g_{12}$ of $q_{12}^{ \pm}$:

$$
q_{12 \alpha_{d}}^{ \pm}\left[f_{12}\right] g_{12}=f_{12}^{ \pm} g_{12},
$$

i) is exactly the usual Fréchet derivative $\tilde{q}_{12}^{ \pm}\left[f_{12}\right] g_{12}$ of $\tilde{q}_{12}^{ \pm}$.

ii) Corresponds to the usual Fréchet derivative $q^{ \pm}[f] g$ of $q^{ \pm}$:

$$
q^{ \pm}[f] g=f^{ \pm} g=f g \pm g f .
$$

Since the \pm operators in (5.20a), (5.8b), (5.21) and (5.18b) satisfy the same algebraic identities (A.3), then important algebraic properties of the recursion operator $\Phi_{12}$ of the KP equation (like hereditariness) are equivalent to the corresponding properties of the nonlocal recursion operator $\widetilde{\Phi}_{12}(5.11)$ and, even more remarkable, of the matrix recursion operator $\Phi_{12}(5.22)$.

In order to make this connection with the matrix formalism more clear, we observe that the nonlocal problem (5.3) can be obtained taking the $N \rightarrow \infty$ limit of the $N \times N$ matrix one dimensional Schroedinger problem

$$
\underline{W} x+\underline{\underline{q}} \underline{W}=\lambda \underline{W}
$$

where the coefficients of the matrix $\underline{\underline{q}}$ are chosen in the form

$$
(\underline{\underline{q}})_{i j}=q_{i j}(x, t)+a\left(\delta_{i, j+1}-\delta_{i, j-1}\right),
$$

with the obvious prescriptions

$$
q_{i j}(x, t) \underset{N \rightarrow \infty}{\longrightarrow} q\left(x, t, y_{1}, y_{2}\right) ; \quad a\left(\delta_{i, j+1}-\delta_{i, j-1}\right) \underset{N \rightarrow \infty}{\longrightarrow} \alpha \frac{\partial}{\partial y_{1}} .
$$

The connection between equations in $2+1$ and $N \times N$ matrix equations in $1+1$ was first used by P. Caudrey. He introduced in [16] a $N \times N$ spectral problem 
(similar to (5.25)) which reduces to (5.2) in the limit $N \rightarrow \infty$. Then he showed that the $N \times N$ Riemann-Hilbert formalism associated with it becomes, in the limit $N \rightarrow \infty$, the nonlocal Riemann-Hilbert and the $\bar{\partial}$ formalisms of (5.2) [17].

The connection established in this section between the spectral problems (5.25), (5.3) and (5.2) implies that the well established theory of recursion operators and their connection to the bi-Hamiltonian formalism in $1+1$ dimensions, once applied to the matrix problem (5.25), gives rise, in the limit $N \rightarrow \infty$, to the corresponding theory developed in [1] and this paper for $2+1$ dimensional systems.

It is remarkable that both algebraic properties and methods of solution for integrable systems in $2+1$ dimensions can be justified and obtained from the corresponding properties of $1+1$ dimensional systems.

\section{Appendix A}

In this Appendix we present some of the explicit calculations necessary to apply the results presented in this paper to the classes of evolution equations associated with the KP and the DS equations. In order to make this paper self-contained, we first present some results contained in Appendices B, C of [1].

The directional derivatives of the basic operators $q_{12}^{ \pm}$and $Q_{12}^{ \pm}$, defined in (1.4b) and (1.10b) respectively, are

$$
\begin{aligned}
& q_{12_{d}}^{ \pm}\left[f_{12}\right] g_{12}=f_{12}^{ \pm} g_{12}, \quad f_{12}, g_{12} \text { scalars, } \\
& Q_{12_{d}}^{ \pm}\left[f_{12}\right] g_{12}=f_{\frac{12}{2}}^{ \pm} g_{12}, \quad f_{12} \text { off-diagonal matrix, }
\end{aligned}
$$

where $f_{12}^{ \pm}$are defined by

$$
f_{12}^{ \pm} g_{12} \doteqdot \int_{\mathbb{R}} d y_{3}\left(f_{13} g_{32} \pm g_{13} f_{32}\right) .
$$

The integral operators $f_{12}^{ \pm}$have the following algebraic properties:

$$
\begin{gathered}
a_{12}^{ \pm} b_{12}= \pm b_{12}^{ \pm} a_{12}, \\
\left(a_{12}^{ \pm} b_{12}^{ \pm}-b_{12}^{ \pm} a_{12}^{ \pm}\right) c_{12}=\left(a_{12}^{-} b_{12}\right)^{-} c_{12}=-c_{12}^{-} a_{12}^{-} b_{12}, \\
\left(a_{12}^{+} b_{12}^{-} \mp b_{12}^{\mp} a_{12}^{ \pm}\right) c_{12}=\left(a_{12}^{\mp} b_{12}\right)^{ \pm} c_{12}= \pm c_{12}^{ \pm} a_{12}^{\mp} b_{12}, \\
a_{12}^{ \pm *}= \pm a_{12}^{ \pm} .
\end{gathered}
$$

Moreover the integral representations

$$
\begin{array}{ll}
q_{12}^{ \pm} f_{12}=\int_{\mathbb{B}} d y_{3}\left(q_{13} f_{32} \pm f_{13} q_{32}\right), & q_{12}=\delta_{12} q_{1}+\alpha \delta_{12}^{\prime}, \\
Q_{12}^{ \pm} f_{12}=\int_{\mathbb{R}} d y_{3}\left(Q_{13} f_{32} \pm f_{13} Q_{32}\right), & Q_{12}=\delta_{12} Q_{1},
\end{array}
$$

imply that the operators $q_{12}^{ \pm}$and $Q_{12}^{ \pm}$satisfy Eqs. (A.3) as well. Equations (A.3) are conveniently used to show that:

a) The recursion operators $\Phi_{12}(1.4)$ and (1.10) are strong symmetries of the starting symmetries $\hat{K}_{12}^{0} H_{12}(1.5-6)$ and $(1.11-12)$ respectively. For example, if 
$\hat{K}_{12}^{0}=Q_{12}^{-}$and $H_{12}$ is given by (1.12),

$$
\begin{aligned}
\Phi_{12 d} & {\left[Q_{12}^{-} H_{12}\right] f_{12}-\left(Q_{12}^{-} H_{12}\right)_{d}\left[\Phi_{12} f_{12}\right]+\phi_{12}\left(Q_{12}^{-} H_{12}\right)_{d}\left[f_{12}\right] } \\
= & -\sigma\left[\left(Q_{12}^{-} H_{12}\right)^{+} P_{12}^{-1} Q_{12}^{+}+Q_{12}^{+} P_{12}^{-1}\left(Q_{12}^{-} H_{12}\right)^{+}\right] f_{12} \\
& \quad-\left(\sigma\left(P_{12}-Q_{12}^{+} P_{12}^{-1} Q_{12}^{+}\right) f_{12}\right)^{-} H_{12}+\sigma\left(P_{12}-Q_{12}^{+} P_{12}^{-1} Q_{12}^{+}\right) f_{12}^{-} H_{12}=0
\end{aligned}
$$

since the terms without $Q_{12}^{ \pm}$give

$$
-\sigma\left(P_{12} f_{12}\right)^{-} H_{12}+\sigma P_{12} f_{12}^{-} H_{12}=0
$$

and the terms with $Q_{12}^{ \pm}$give

$$
\begin{aligned}
-\sigma( & \left.Q_{12}^{-} H_{12}\right)^{+} P_{12}^{-1} Q_{12}^{+} f_{12}-\sigma Q_{12}^{+} P_{12}^{-1}\left(Q_{12}^{-} H_{12}\right)^{+} f_{12} \\
& +\left(\sigma Q_{12}^{+} P_{12}^{-1} Q_{12}^{+} f_{12}\right)^{-} H_{12}-\sigma Q_{12}^{+} P_{12}^{-1} Q_{12}^{+} f_{12}^{-} H_{12}=-\sigma\left(\left(\left(Q_{12}^{-} H_{12}\right)^{+}\right.\right. \\
& \left.\left.+H_{12}^{-} Q_{12}^{+}\right) P_{12}^{-1} Q_{12}^{+} f_{12}+Q_{12}^{+} P_{12}^{-1}\left(f_{12}^{+} Q_{12}^{-} H_{12}+Q_{12}^{+} f_{12}^{-} H_{12}\right)\right) \\
= & -\sigma Q_{12}^{+} P_{12}^{-1}\left(H_{12}^{-} Q_{12}^{+} f_{12}+f_{12}^{+} Q_{12}^{-} H_{12}+Q_{12}^{+} f_{12}^{-} H_{12}\right)=0 .
\end{aligned}
$$

b) The Lie algebra of the starting symmetries is given by Eqs. (1.7) and (1.13).

\section{For example}

i) if $\hat{K}_{12}^{0} H_{12}$ are given by $(1.5-6)$ :

$$
\begin{aligned}
{\left[\hat{N}_{12} H_{12}^{(1)}, \hat{M}_{12} H_{12}^{(2)}\right]_{d}=} & \left(\left(D q_{12}^{+}+q_{12}^{-} D^{-1} q_{12}^{-}\right) H_{12}^{(2)}\right)^{-} H_{12}^{(1)}-D\left(q_{12}^{-} H_{12}^{(1)}\right)^{+} H_{12}^{(2)} \\
& -\left(q_{12}^{-} H_{12}^{(1)}\right)^{-} D^{-1} q_{12}^{-} H_{12}^{(2)}-q_{12}^{-} D^{-1}\left(q_{12}^{-} H_{12}^{(1)}\right)^{-} H_{12}^{(2)} \\
= & -D q_{12}^{+}\left(H_{12}^{(1)}\right)^{-} H_{12}^{(2)}+q_{12}^{-} D^{-1}\left(-\left(H_{12}^{(1)}\right)^{-} q_{12}^{-} H_{12}^{(2)}\right. \\
& \left.+\left(H_{12}^{(2)}\right)^{-} q_{12}^{-} H_{12}^{(1)}\right)=-\hat{M}_{12} H_{12}^{(3)} ;
\end{aligned}
$$

ii) if $\hat{K}_{12}^{0} H_{12}$ are given by $(1.11-12)$

$$
\begin{aligned}
{\left[\hat{N}_{12} H_{12}^{(1)}, \hat{M}_{12} H_{12}^{(2)}\right]_{d} } & =\left(Q_{12}^{-} \sigma H_{12}^{(2)}\right)^{-} H_{12}^{(1)}-\left(Q_{12}^{-} H_{12}^{(1)}\right)^{-} \sigma H_{12}^{(2)} \\
& =-\left(H_{12}^{(1)}\right)^{-} Q_{12}^{-} \sigma H_{12}^{(2)}+\left(\sigma\left(H_{12}^{(2)}\right)\right)^{-} Q_{12}^{-} H_{12}^{(1)} \\
& =-\hat{M}_{12} H_{12}^{(3)} .
\end{aligned}
$$

c) The functions $T_{12}$ given by (4.16) and (4.25) satisfy Eqs. (4.10); for examples

i) if $T_{12}=\delta_{12}$, then

$$
S_{12} f_{12}=\Phi_{12 d}\left[\delta_{12}\right] f_{12}=\left(2 \delta_{12}^{+}+\delta_{12}^{-} D^{-1} q_{12}^{-}+q_{12}^{-} D^{-1} \delta_{12}^{-}\right) f_{12}=4 f_{12},
$$

since $\delta_{12 d}=0$ and $\delta_{12}^{+} f_{12}=2 f_{12}, \delta_{12}^{-} f_{12}=0$.

ii) If $T_{12}=(x / 2) \sigma Q_{12}^{+} \delta_{12} I$, then Eqs. (4.10) are satisfied using the following results:

$$
\begin{gathered}
T_{12 d}\left[f_{12}\right]=\frac{x}{2} \sigma f_{12}^{+} \delta_{12} I=x \sigma f_{12}, \\
T_{12}^{ \pm} f_{12}=x\left(\sigma Q_{1} f_{12} \pm f_{12} \sigma Q_{2}\right)=x \sigma \begin{cases}Q_{12}^{ \pm} f_{12}, & f_{12} \text { off-diagonal } \\
Q_{12}^{ \pm} f_{12}, & f_{12} \text { diagonal }\end{cases}
\end{gathered}
$$

For instance:

$$
\begin{aligned}
S_{12} f_{12}= & -\sigma\left(T_{12}^{+} P_{12}^{-1} Q_{12}^{+}+Q_{12}^{+} P_{12}^{-1} T_{12}^{+}\right) f_{12}+\sigma\left(P_{12}-Q_{12}^{+} P_{12}^{-1} Q_{12}^{+}\right) x \sigma f_{12} \\
& -x\left(P_{12}-Q_{12}^{+} P_{12}^{-1} Q_{12}^{+}\right) f_{12}=f_{12} .
\end{aligned}
$$


d) $\Phi_{12}^{n}\left[\hat{K}_{12}^{0} H_{12}, T_{12}\right]=0$, if $\hat{K}_{12}^{0} H_{12}$ and $T_{12}$ are given by (1.11-12) and (4.25) respectively, or by $q_{12}^{-} H_{12}, H_{12}=H\left(y_{1}, y_{2}\right)$, and $\delta_{12}$. For example

i) $\Phi_{12}^{n}\left[q_{12}^{-} H_{12}, \delta_{12}\right]_{d}=\Phi_{12}^{n} \delta_{12}^{-} \cdot H_{12}=0$.

ii) $\Phi_{12}^{n}\left[Q_{12}^{-} H_{12}, T_{12}\right]_{d}=\Phi_{12}^{n}\left(T_{12}^{-} H_{12}-T_{12_{d}}\left[Q_{12}^{-} H_{12}\right]\right)$

$$
=\Phi_{12}^{n}\left(x \sigma Q_{12}^{-}-x \sigma Q_{12}^{-}\right) \cdot H_{12}=0 \text {. }
$$

e) Equation (4.17b) holds. It follows from $\hat{M}_{12 d}\left[\delta_{12}\right]=D \delta_{12}^{+}+\delta_{12}^{-} D^{-1} q_{12}^{-}+$ $q_{12}^{-} D^{-1} \delta_{12}^{-}=2 D$, which implies

$$
\Phi_{12}^{n}\left[\hat{M}_{12} \cdot H_{12}, \delta_{12}\right]_{d}=2 \Phi_{12}^{n} D \cdot H_{12} .
$$

Different choices of $H_{12}=H\left(y_{1}, y_{2}\right)$ give different results. As it was shown in Appendix B of [1]

$$
\Phi_{12}^{n+1} D \cdot H_{12}=\sum_{l=0}^{n}(2 \alpha)^{l} \Phi_{12}^{n-l} \hat{M}_{12} \cdot H_{12}^{(l)}, \quad H_{12}^{(l)} \doteqdot \partial^{l} \frac{H\left(y_{1}-y_{2}\right)}{\partial y_{1}^{l}} ;
$$

an analogous, although more tedious derivation, gives

$$
\begin{gathered}
\Phi_{12}^{n+1} D \cdot H_{12}=\Phi_{12}^{n} \hat{M}_{12} \cdot H_{12}+\sum_{l=1}^{v_{n}} a_{l}(2 \alpha)^{2 l} \Phi_{12}^{n-2 l} \hat{M}_{12} \cdot H_{12}^{(2 l)}, \\
H_{12}^{(l)} \doteqdot \frac{\partial^{l} H\left(y_{1}+y_{2}\right)}{\partial y_{1}^{l}}, \quad a_{l} \doteqdot C_{l}^{(l)}, \quad v_{n} \doteqdot \begin{cases}(n-1) / 2, & n \text { odd } \\
n / 2, & n \text { even }\end{cases}
\end{gathered}
$$

and the coefficients $C_{l}^{(l)}$ are obtained through the following recursive construction:

$$
\begin{aligned}
& C_{l}^{(m)}=C_{l}^{(m-1)}+2 C_{l-1}^{(m-1)}+C_{l-2}^{(m-1)}, \\
& C_{0}^{(0)}=1,
\end{aligned}
$$

where $C_{b}^{(a)}=0$ if $b<0$ and $b>a$. Equations (A.4) and (A.6) imply Eq. (4.17b).

f) $\Theta_{12}^{-1} \Phi_{12}^{n} \hat{K}_{12}^{0} H_{12}$ are extended gradients; for example if

i) $\hat{K}_{12}^{0}=\hat{N}_{12} \doteqdot q_{12}^{-}, H_{12}=H\left(y_{1}, y_{2}\right), \Theta_{12}=D$ and $n=0$ :

$$
\begin{aligned}
\left\langle f_{12},\left(D^{-1} \hat{N}_{12} H_{12}\right)_{d}\left[g_{12}\right]\right\rangle & =\left\langle f_{12}, D^{-1} g_{12}^{-} H_{12}\right\rangle=\left\langle D^{-1} f_{12}, H_{12}^{-} g_{12}\right\rangle \\
& =-\left\langle H_{12}^{-} D^{-1} f_{12}, g_{12}\right\rangle=\left\langle D^{-1} f_{12}^{-} H_{12}, g_{12}\right\rangle .
\end{aligned}
$$

ii) $\hat{K}_{12}^{0}=\hat{M}_{12} \doteqdot D q_{12}^{+}+q_{12}^{-} D^{-1} q_{12}^{-}, H_{12}=H\left(y_{1}, y_{2}\right), \Theta_{12}=D$ and $n=0$ :

$$
\begin{aligned}
\left\langle f_{12},\left(D^{-1} \hat{M}_{12} H_{12}\right)_{d}\left[g_{12}\right]\right\rangle & \\
& =\left\langle f_{12}, g_{12}^{+} H_{12}+D^{-1} g_{12}^{-} D^{-1} q_{12}^{-} H_{12}+D^{-1} q_{12}^{-} D^{-1} g_{12}^{-} H_{12}\right\rangle \\
& =\left\langle f_{12},\left(H_{12}^{+}-D^{-1}\left(\left(D^{-1} q_{12}^{-} H_{12}\right)^{-}+q_{12}^{-} D^{-1} H_{12}^{-}\right)\right) g_{12}\right\rangle \\
& =\left\langle\left(H_{12}^{+}-\left(\left(D^{-1} q_{12}^{-} H_{12}\right)^{-}+H_{12}^{-} D^{-1} q_{12}^{-}\right) D^{-1}\right) f_{12}, g_{12}\right\rangle \\
& =\left\langle\left(H_{12}^{+}-D^{-1}\left(\left(D^{-1} q_{12}^{-} H_{12}\right)^{-}+q_{12}^{-} H_{12}^{-} D^{-1}\right)\right) f_{12}, g_{12}\right\rangle .
\end{aligned}
$$

iii) $\hat{K}_{12}^{0}=\hat{M}_{12} \doteqdot Q_{12}^{-} \sigma, H_{12}$ defined in (1.12) and $n=0$ :

$$
\left\langle f_{12},\left(\sigma \hat{M}_{12} H_{12}\right)_{d} g_{12}\right\rangle=\left\langle f_{12},-H_{12}^{+} g_{12}\right\rangle=\left\langle-H_{12}^{+} f_{12}, g_{12}\right\rangle \text {. }
$$

iv) $\hat{K}_{12}^{0}=\hat{N}_{12} \doteqdot Q_{12}^{-}, H_{12}$ defined in (1.12) and $n=1$ : 


$$
\begin{aligned}
& \left\langle f_{12},\left(\sigma \hat{N}_{12}^{(1)} H_{12}\right)_{d} g_{12}\right\rangle \\
& \quad=\left\langle f_{12},\left(-\left(P_{12}^{-1} Q_{12}^{+} Q_{12}^{-} H_{12}\right)^{+}-P_{12} H_{12}^{-}+Q_{12}^{+} H_{12}^{-} P_{12}^{-1} Q_{12}^{+}\right) g_{12}\right\rangle \\
& \quad=\left\langle\left(-\left(P_{12}^{-1} Q_{12}^{+} Q_{12}^{-} H_{12}\right)^{+}-P_{12} H_{12}^{-}+Q_{12}^{+} H_{12}^{-} P_{12}^{-1} Q_{12}^{+}\right) f_{12}, g_{12}\right\rangle .
\end{aligned}
$$

g) Equation (4.24b) holds, since

$$
\begin{aligned}
\left\langle\hat{\gamma}_{12}^{(n+1)} H_{12}, x \sigma Q_{12}^{+} \delta_{12} I\right\rangle & =-\left\langle x Q_{12}^{+} \sigma \hat{\gamma}_{12}^{(n+1)} H_{12}, \delta_{12} I\right\rangle \\
& =\int_{\mathbb{R}^{3}} d x d y_{1} d y_{2} \delta_{12} \operatorname{trace} Q_{12}^{+} \sigma \hat{\gamma}_{12}^{(n+1)} H_{12} .
\end{aligned}
$$

\section{Appendix B}

In this Appendix we show that if $\Phi$ is factorizable in terms of compatible Hamiltonian operators $\Omega$ and $\Theta$ in the form $\Phi=\Omega \Theta^{-1}$, and if $\Theta$ is invertible and $\Theta_{L}=0$, then Eq. (4.5) holds.

We first show that

$$
\begin{gathered}
(\Phi T)_{L}^{*}=\mathscr{L}_{T}^{*}+T_{L}^{*} \Phi^{*}, \quad \mathscr{L}_{T} b \doteqdot \Phi_{L}[b] T, \\
\Phi_{L}[v] T+\Theta \mathscr{L}_{T}^{*} \Theta^{-1} v=\Phi_{L}[T] b .
\end{gathered}
$$

(B.1) simply follows from the definition of the adjoint:

$$
\left\langle(\Phi T)_{L}^{*} a, b\right\rangle=\left\langle a, \Phi_{L}[b] T+\Phi T_{L}[b]\right\rangle=\left\langle\left(\mathscr{L}_{T}^{*}+T_{L}^{*} \Phi^{*}\right) a, b\right\rangle,
$$

while (B.2) requires the use of all the hypothesis of this Lemma.:

$$
\begin{aligned}
& \left\langle\Phi_{L}[v] T+\Theta \mathscr{L}_{T}^{*} \Theta^{-1} v, \alpha\right\rangle \\
& \quad=\left\langle\Omega_{L}\left[\Theta\left(\Theta^{-1} v\right)\right] \Theta^{-1} T, \alpha\right\rangle+\left\langle\Omega_{L}[\Theta \alpha] \Theta^{-1} v, \Theta^{-1} T\right\rangle \\
& \quad=\left\langle\alpha, \Omega_{L}[T] \Theta^{-1} v\right\rangle=\left\langle\alpha, \Phi_{L}[T] v\right\rangle .
\end{aligned}
$$

Then, using (B.1-2) and (4.4) for $n=0$, we obtain Eq. (4.5):

$$
\begin{aligned}
\left((\Phi T)_{L}+\Theta(\Phi T)_{L}^{*} \Theta^{-1}\right) v= & \Phi\left(T_{L}[v]+\Theta T_{L}^{*} \Theta^{-1} v\right)+\Phi_{L}[v] T \\
& +\Theta \mathscr{L}_{T}^{*} \Theta^{-1} v+\Theta\left(T_{L}^{*} \Phi^{*}-\Phi^{*} T_{L}^{*}\right) \Theta^{-1} v \\
= & \Phi\left(T_{L}[v]+\Theta T_{L}^{*} \Theta^{-1} v\right)+\Theta S^{*} \Theta^{-1} v .
\end{aligned}
$$

Acknowledgements. It is a pleasure to acknowledge useful discussions with M. J. Ablowitz. One of the authors (P.M.S.) wishes to acknolwedge the warm hospitality of the Mathematics Department of the University of Paderborn, where the last part of this paper was completed. He also acknowledges interesting discussions with B. Fuchssteiner and W. Oevel. Particular thanks go to W. Oevel for generously computing some of the functions $\Phi_{12}^{m} \hat{K}_{12}^{0} H_{12}^{(r)}$ discussed in this paper, using the system of algebraic manipulation MACSYMA. This work was supported by the National Science Foundation under grant number DMS 8501325 and the Office of Naval Research under grant number N00014-76-C-0867.

\section{References}

1. Santini, P. M., Fokas, A. S.: Recursion operators and bi-Hamiltonian structures in multidimension I. Commun. Math. Phys. 115, 375-419(1988)

2. Magri, F.: A simple model of the integrable Hamiltonian equation. J. Math. Phys. 19, 1156 (1978); 
Nonlinear evolution equations and dynamical systems. Boiti, M., Pempinelli, F., Soliani, G. (eds.): Lecture Notes in Physics Vol. 120, p. 233 Berlin, Heidelberg New York: Springer 1980

3. Gel'fand, I. M., Dorfman, I. Y.: Funct. Anal. Appl. 13, 13 (1979); 14, 71 (1980)

4. Fokas, A. S., Fuchssteiner, B.: On the structure of symplectic operators and hereditary symmetries. Lett. Nuovo Cimento 28, 299 (1980); Fuchssteiner, B., Fokas, A. S.: Symplectic structures, their Bäcklund transformations and hereditary symmetries, Physica 4D, 47 (1981)

5. Fokas, A. S., Fuchssteiner, B.: The hierarchy of the Benjamin-Ono equation. Phys. Lett. A86, 341 (1981)

6. Oevel, W., Fuchssteiner, B.: Explicit formulas for symmetries and conservation laws of the Kadomtsev-Petriashvili equations. Phys. Lett. A88, 323 (1982)

7. Chen, H. H., Lee, Y. C., Lin, J. E.: Physica 9D, 493 (1983)

8. Chen, H. H., Lin, J. E., Lee, Y. C.: On the direct construction of the inverse scattering operators of nonlinear Hamiltonian systems (preprint) 1986

9. Oevel, W.: A geometrical approach to integrable systems admitting scaling symmetries. (Preprint) University of Paderborn, January 1986

10. Magri, F.: (private communication)

11. Fuchssteiner, B.: Mastersymmetries, higher order time dependent symmetries and conserved densities of nonlinear evolution equations. Prog. Theor. Phys. 70, 1508 (1983)

12. Dorfman, I. Y.: Deformations of the Hamiltonian structures and integrable systems (preprint)

13. Oevel, W.: Mastersymmetries for finite dimensional integral systems: The Calogero-Moser system. Preprint, University of Paderborn (1986)

14. Fokas, A. S., Santini, P. M.: Stud. Appl. Math. 75, 179 (1986)

15. Calogero, F., Degasperis, A. Nonlinear evolution equations solvable by the inverse spectral transform. II. Nuovo Cim. 39B, 1 (1977)

16. Caudrey, P.: Discrete and periodic spectral transforms related to the Kadomtsev-Petviashvili equation. Preprint U.M.I.S.T.

17. Fokas, A. S., Ablowitz, M. J.: Stud. Appl. Math. 69, 211 (1983); Ablowitz, M. J., BarYaacov, D., Fokas, A. S.: Stud. Appl. Math. 69, 135 (1983)

18. Case, K. M.: Theorem on linearized Hamiltonian systems. J. Math. Phys. 26, 1201 (1985)

19. David, D., Kamran, N., Levi, D., Winternitz, P.: Symmetry reduction for the KP equation using a loop algebra. (Preprint), Université De Montréal (1985)

20. Yu. Orlof, A., Schulman, E. I.: Additional symmetries for integrable equations and conformal algebra representation. Lett. Math. Phys. 12, 171 (1986)

Communicated by A. Jaffe

Received December 30, 1986; in revised form August 10, 1987 